\title{
Analysis of Star Formation in Galaxy-like Objects
}

\author{
Patricia B. Tissera ${ }^{1}$
}

Received —

1 I.A.F.E., Casilla de Correos 67, Suc. 28, Buenos Aires (1428), Argentina. E-mail: patricia@iafe.uba.ar 


\begin{abstract}
Using cosmological hydrodynamical simulations, we investigate the effects of hierarchical aggregation on the triggering of star formation in galactic-like objects. We include a simple star formation model to transform the cold gas in dense regions into stars. Simulations with different parameters have been performed in order to quantify the dependence of the results on the parameters. We then resort to stellar population synthesis models to trace the color evolution of each object with red-shift and in relation to their merger histories. We find that, in a hierarchical clustering scenario, the process of assembling of the structure is one natural mechanism that may trigger star formation. The resulting star formation rate history for each individual galactic object is composed of a continuous one $\left(\leq 3 \mathrm{M}_{\odot} / \mathrm{yr}\right)$ and a series of star bursts. We find that even the accretion of a small satellite can be correlated with a stellar burst. Massive mergers are found to be more efficient at transforming gas into stars
\end{abstract}

Subject headings: galaxies: evolution - galaxies: formation - galaxies: interactions galaxies: fundamental parameters - cosmology: theory - cosmology: dark matter 


\section{Introduction}

Within the last few years, it has been possible to start drawing an observational picture of the formation and evolution of galaxies (e.g. Fukigita et al. 1996) based upon an unprecedented amount of results on the astrophysical properties of galactic objects up to $z \approx 5$ at ultraviolet, optical and near IR wavelengths (e.g. Steidel \& Hamilton 1992; Madau 1995; Lilly et al. 1995; Cowie et al. 1996; Ellis et al. 1996; Steidel et al. 1988). These data provide observational constraints on the history of structure formation in the Universe. In particular, there has been a significant progress in describing the cosmic history of star formation and metal enrichment (e.g. Madau et al. 1996; Lowenthal et al. 1997). Hopefully these data together with previous and future results will contribute to the understanding of the process of star formation. Although there are several works on this subject in the scientific literature (e.g. Kennicutt 1996; Ferrini 1997 and references therein; Kennicutt 1998), the detailed physical mechanism is still under study since several aspects remain poorly understood. One open question is regarding the factors that trigger and control the star formation activity in galaxies of different morphologies.

In a hierarchical clustering model where structure forms in a bottom-up way, a typical galactic halo is the result of the mergers of smaller substructures. The halo merger frequency and the typical mass involved in one event vary depending on the environment and the cosmological model assumed, among other factors. The astrophysical properties of the galactic objects are determined by all these processes and by the hydro-dynamical evolution of the baryons in a complex way. The conditions which may trigger and control the transformation of cold gas in dense regions into stars are not yet well understood and may involve different processes such as supernova energy injection, mergers, disk instabilities and tidal fields. Of particular interest is the possible triggering of star formation by the merging of substructures as a galactic object is assembled in a hierarchical clustering scenario. This fact would imply the existence of a correlation between mergers with satellites and enhancements of the star formation activity. Hereafter, we will define a merger as the complete process since the time two baryonic objects are identified to share the same dark matter halo for the first time to their actual fusion. And whenever tidal fields are mentioned, they 
are those originated during the mergers. The study of any interactions that do not result in the actual fusion of the structures is not considered in this paper.

There has been also much work on the link between the high star formation rates in ultra-luminous IRAS galaxies (ULIRGs) and their environments, although a consensus on how the galaxy environment influences its star formation rate has yet to be reached. Lawrence et al. (1989) find that most ( 70\%) of the ULIRGS they studied appeared to have close companions or be morphologically disturbed (Lucas et al. 1997; see also Sanders \& Mirabel 1996), whilst a significant minority appear to be isolated. Other authors such as Clements et al. (1996) and Sanders et al. (1988) estimate that $~ 99 \%$ of ULIRGs are in interacting systems. It is clear that much of the discrepancy is due to the difficulty in classifying quantitatively what is meant by 'interacting'. Despite this reliance on a rather subjective classification of the data, there seems to be a trend in increasing likelihood of interaction and increasing starburst luminosity in IRAS selected galaxies. All the above studies of the environments of star-bursting galaxies assume that both, the environmental trigger and the starburst episode, are contemporaneous. As Joseph and Wright (1985) point out, this might not be true if we use tidal tails as the evidence for an interaction as, depending on the stellar initial mass function (IMF), the tidal tail might last longer than the starburst it triggers. Therefore it is still unclear as to whether an interaction/merger is required in order for a starburst to take place and also whether they happen simultaneously.

On the other hand, recent observations (mainly from the Hubble Deep Field) show an increasing number of irregular/interacting or morphologically disturbed objects with look-back time, which also exhibit strong star formation activity (e.g. Glazebrook et al. 1995; Ellis et al. 1996; Lilly et al. 1997; Lowenthal et al. 1997; Guzmán et al. 1997; Driver et al. 1998). It is not yet clear at what extent these data are fully consistent with predictions of models based on hierarchical clustering. Nevertheless all of them agree in supporting a scenario where interactions/mergers and star formation activity increase with red-shift (e.g. Bouwens et al. 1997; Driver et al. 1998; Brichmann et al. 1998).

From a theoretical point of view, semi-analytic models have been quite successful in 
formulating a picture of galaxy formation and evolution, although they have to resort to global recipes in order to take into account complex effects (Kauffmann et al. 1993; Baugh et al. 1996). In this regard, Lacey \& Silk (1991) have also used tidal interactions between galaxy-sized objects for controlling the star formation processes in galactic objects, although they did not consider mergers of substructure as a possible cause. Baugh et al. (1996) formulate a semi-analytic model where the morphology of a galaxy is determined by its history of major mergers which trigger violent star formation. No predictions for the strength, duration and frequency of these stellar bursts could be done with this model.

Self-consistent numerical simulations have proved a powerful tool for the study of the formation of galaxies in a cosmological framework (Cen et al. 1998; Hernquist et al. 1996; Theuns et al. 1998; Tissera et al. 1997; Pearce et al. 1999; Katz et al. 1999). They have the advantage over semi-analytic models of being able to provide a consistent description of the evolution of the structure in the non-linear regime. As a consequence, physical processes related to the evolution of the dissipative component can be included and modeled upon a more physical basis. Among these processes, star formation mechanisms are highly important because of their outstanding role in the formation of structure on galactic scales. However, the treatment of processes related with star formation is at its beginnings, since numerical problems, added to the lack of a fully theoretical understanding, make its implementation difficult. Several authors have analyzed numerical models of the formation of individual galaxies including simple schemes to transform the cold dense gas into stars (e.g. Katz 1992; Navarro \& White, Gerritsen 1997).

Of most relevance to the analysis carried out in this paper are the results of Barnes \& Hernquist $(1991,1996)$ and Mihos \& Hernquist $(1994,1996)$. These authors modeled mergers of two disk/halo galaxies using hydro-dynamical codes. They found that a merger with a satellite can induce the formation of a bar along which the gas is compressed and shocked, loosing angular momentum. This process would trigger a gas inflow which could fuel star formation activity at the center. These models illustrate one way in which tidal fields can produce gas inflows in strongly interacting and merging galaxies. Mihos and Hernquist (1996) also showed that the internal 
structure of a parent disk-like galaxy is relevant to regulate the rate of this gas inflow. Hernquist (1989 a,b) showed that even the accretion of low-mass satellites by disks can result in an inward gas flow. In all cases, by applying the Schmidt law, an enhancement of the gas density directly implies an increase of the star formation activity.

With the models described in this work, we intend to establish the relevant characteristics of the interplay between hierarchical aggregation and star formation, and to assess if the outcomings are consistent with observations of galaxies undergoing star formation activity in a cosmological framework. Note, however, that we intend to analyze the star formation process in normal field galactic-like objects. We will refer to mergers that arise as a consequence of their formation and evolution in a hierarchical cosmological scenario. In hierarchical clustering scenarios, galactic objects build up through the aggregation of substructure and may suffer major and minor encounters throughout their life. Their star formation histories may be affected by these mergers and, as already pointed out by several authors (e.g., Mihos \& Hernquist 1996, Barnes \& Hernquist 1996; Baugh et al. 1996), they may contribute to its triggering. As an attempt to assess the relation between this hierarchical built-up of the structure and the star formation process, we analyze the evolutionary history of each halo in our simulations, looking for possible correlations between star formation enhancements and mergers. The main difference between previous works and this one, is that, in this case, every merger event arises naturally, in consistency with a cosmological model. In fully consistent cosmological simulations, the distribution of merger parameters, such as the orbit characteristics, the orbital energy and angular momentum, the masses of the virial halos and baryonic clumps involved in the merger event, and the spin, internal structure and relative orientation of the baryonic clumps that are about to merge, among others, arise naturally at each epoch as a consequence of the initial spectrum of the density fluctuation field, its normalization, and the cosmological models and its parameters. In controlled type mergers, they are set by hand. Moreover, in fully-consistent cosmological simulations, the effects of diffuse gas accretion and of interactions with small satellites on the assembly of a galactic object are also accounted for. 
We will also intend to know at what extent can a simple star formation (SF) model follow the SF history of a galaxy-like object, if those SF histories are consistent with observations and how sensitive they are to the free parameters of the model. We will also use the technique described by Tissera, Lambas \& Abadi (1997) to assign luminosities at different wavelengths to the simulated galactic objects. This implementation allows us to follow the evolution of their colors as a function of $z$ and in relation to their evolutionary history.

This paper is organized as follows. Section 2 describes the main characteristics and parameters of the simulations. Section 3 analyses the results and compares them with recent observational data. In Section 4 we investigate the effects of mergers on the color distributions of the galaxies formed, and Section 5 outlines the results.

\section{Simulations}

The simulations analyzed follow the evolution of a typical region of the Universe using a version of AP3M+SPH code (Tissera et al. 1997). We carried out three simulations (hereafter referred as S.1, S.2 and S.3). S.1 and S.2 share the same initial conditions for the distributions of gas and dark matter particles, whilst S.3 has different ones. Simulations S.1 and S.2 are identical except for the star formation efficiency, so any difference between them is due to the SF process and the fact that stars behave as collision-less particles.

The initial conditions are set up by using ACTION (for S.1 and S.2) and COSMICS (for S.3), and are consistent with a Cold Dark Matter (CDM) spectrum with $\Omega=1, \Lambda=0, \Omega_{\mathrm{b}}=0.1$, and $\sigma_{8}=0.4$ for S.1, S.2 and $\sigma_{8}=0.67$ for S.3. We used $N=262144$ particles $\left(\mathrm{N}_{\text {dark }}=259520\right.$ and $\left.\mathrm{N}_{\mathrm{bar}}=26214\right)$ in a comoving box of length $L=5 h^{-1} \mathrm{Mpc}\left(H_{0}=100 h^{-1} \mathrm{~km} \mathrm{~s}^{-1} \mathrm{Mpc}^{-1}, h=0.5\right)$. Note that dark matter and baryonic particles have the same mass $\left(\mathrm{M}_{\text {part }}=2.6 \times 10^{8} \mathrm{M}_{\odot}\right)$. The gravitational softening used in these simulations is $3 \mathrm{kpc}$, and the smaller smoothing length allowed is $1.5 \mathrm{kpc}$. The time-steps of integration used are $\Delta t=1.4 \times 10^{7} \mathrm{yr}$ for S.1 and S.2, and $\Delta t=1.2 \times 10^{7} \mathrm{yr}$ for S.3. These simulations have proved to be adequate to study processes related 
with formation of galaxies in a fully cosmological framework (e.g., Tissera et al. 1997; Tissera \& Domínguez-Tenreiro 1998; Domínguez-Tenreiro et al. 1998; Tissera, Sáiz \& Domínguez-Tenreiro 1999) despite they have lower hydro-dynamical resolution when compared to prepared ones (Navarro \& Steinmetz 1997).

These simulations include star formation according to the algorithm described by Tissera et al. 1997. The gas cools due to radiative cooling. We use the approximation for the cooling function given by Dalgarno and McCray (1972). Gas particles are transformed into stars if they are cold $\left(T_{*} \leq 3 \times 10^{4} \mathrm{~K}\right.$ for S.1 and S.2, and $T_{*} \leq 10^{4} \mathrm{~K}$ for S.3 $)$, dense $\left(\rho>\rho_{\text {crit }} \approx 7 \times 10^{-26} \mathrm{gr} \mathrm{cm}^{-3}\right)$ and satisfy the Jean's instability criterium. When a gas particle satisfies these conditions, it is transformed into a star particle after a time interval $(\tau)]^{2}$ over which its gas mass is being converted into stars according to

$$
\frac{d \rho_{\mathrm{star}}}{d t}=-c \frac{\rho_{\mathrm{gas}}}{t_{*}}
$$

where $c$ is the star formation efficiency $(c=0.01,0.1,0.01$ for S.1, S.2 and S.3, respectively) and $t_{*}$ is a characteristic time-scale assumed to be equal to the dynamical time of the particle $\left(t_{*}=t_{\mathrm{dyn}}=\left(3 \pi /\left(16 G \rho_{\text {gas }}\right)\right)^{1 / 2}\right)$.

The simulations have different star formation efficiency parameters, $c$. The total number of stars formed will depend on the values of $c$ and $T_{*}$, which can be adjusted in order to reproduce observations. Simulations S.1, S.2 and S.3 have transformed 12\%, 28\% and 7.5\%, respectively, of their total baryonic mass into stars at $z=0$. Because of the higher value of $\sigma_{8}$ used in S.3, halos collapse earlier and reach higher densities sooner. As a consequence, stars form from higher $z$ depleting quicker the gas in condition of forming stars. Hence, the SF history depends also on the normalization of the power spectrum (Baugh et al. 1996). Different values of critical temperature

\footnotetext{
2 This time interval is the time estimated from equation (1) over which $99 \%$ of the gas mass in a particle is expected to be transformed into stars (Navarro \& White). It is estimated as: $\tau=\ln 0.01 t_{*} / c$.
} 
$T_{*}$ have been used. Simulation S.3 has a lower value which helps to produce less stars than in S.1. The total stellar masses at $z=0$ imply a stellar density parameter $\Omega_{\mathrm{S}}$ greater than the observed ones: $0.005<\Omega_{\mathrm{s}} h^{2}<0.009$ (Madau 1998), although the latter are subject to serious uncertainties such as dust effects which can lead to important underestimations. Recent observations in the mid and far infrared suggest higher values (Flores et al. 1998).

Note also that we have adopted $\Omega_{\mathrm{b}}=0.10$, a value that could be considered somewhat high. However, recent measurements of the deuterium abundance in clouds of hydrogen at high red-shift (Burles \& Tytler: Burles \& Tytlen), if correct, allow to constrain the baryon fraction to a precision of $10 \%, \Omega_{\mathrm{b}}=0.08 \pm 0.008$ (for $h=0.5$, as we have assumed), close to the value we have used.

Feedback effects and metallicity enrichment by supernova explosions have not been included in these simulations. Feedback processes are believed to play a key role in helping to set a self-regulated star formation regimen (Silk 1997). But its modeling in hydro-dynamical simulations is still quite controversial (Katz 1992; Navarro \& White; Metzler \& Evrard 1995; Yepes et al. 1997). Since the SF process is not completely understood, it is wise to analyze these two effects in separate steps.

One shortcoming of numerical simulations is that numerical resolution decreases with look-back time. The higher the red-shift, the smaller the objects and so, the smaller the number of particles used to resolve them. This is an ubiquitous problem in all numerical simulations and a very large number of particles would be required to improve it. Since this is impossible to accomplish at present, results should always be considered with caution. In order to minimize this problem, we will study the evolutionary history of the larger objects in our simulations (see Section 3). Note, however, that we use fully self-consistent cosmological simulations, hence, the hierarchical evolution of a galactic halo is very well represented and so are mergers and the effects of tidal fields generated by the nearby structure.

\section{Analysis}




\subsection{Global Star Formation}

Recent observations of objects at different $z$ have provided with information about the SF history of the Universe (Somverville \& Primack 1998 an references therein). Although many uncertainties such as the initial mass function, reddening by internal and Ly $\alpha$ cloud absorptions and the fact that UV luminosity traces mainly the formation of massive stars, among others, affect these results, it is possible to envisage a global $\mathrm{SF}$ trend. In this sense, this observational relation can be a useful tool to assess how the SF proceeds within a simulated box. It has to stressed that we do not intend to explain what is observed but to use observations as a global constrain to qualitatively compare the effects that the different SF parameters may have on the SF history of the simulated box.

We estimate the global SFR by reckoning the stellar mass formed in the simulated box at each $z$, and smoothing it overtime in order to diminish the noise introduced by the discreteness of the SF process. In order to compare these mean star formation rate $<S F R>$ histories with observational results, we calculate the cosmic star formation rate density: $\rho_{\mathrm{SFR}}=<S F R>/ \mathrm{V}$, at

each time-step of integration in each simulation. $\mathrm{V}$ is the comoving volume of the simulated boxes at each corresponding $z$.

In Figure 1, we plot $\rho_{S F R}$ for simulations S.1, S.2 and simulation S.3, and include recent observational results. As can be seen from this figure, the simulated $\rho_{S F R}$ are quite different. None of them have a peak at $z \approx 1.5$ as that claimed by Madau et al. (1996), but they are within the observed range.

Simulations S.1 and S.2, because of the combined effects of the lower normalization parameter $\sigma_{8}$ and SF ones, they start forming stars later on. The only difference between S.1 and S.2 is the value of $c$. Therefore, it is direct that a change in the star formation efficiency introduces a delay in process and decreases the overall star formation rates. To fill up the gap between $z \approx 2$ and the last point measured by Madau et al. (1996) at $z=5.5$, it can be estimated that approximately 20 gas particles should have been transformed into stars at a constant rate of $45 \mathrm{M}_{\odot} / \mathrm{yr}$. This 
number represents less than a $10 \%$ of the total stellar mass formed in S.1 and S.2. Whence we can conclude that the results for $z<2$ will not be strongly affected by the necessary changes in the SF process in order to have a complete SFR history at higher $z$.

Simulation S.3 starts forming stars at larger $z$ due to the higher normalization parameter $\sigma_{8}$ adopted. But as a consequence of the low $c$ and $T_{*}$ values used, the $\mathrm{SF}$ rates are lower than those in the other two simulations. Globally, the $\langle S F R>$ in S.3 shows a similar trend to observations. In this simulation, the total amount of stars formed at $z>2$ is $30 \%$ of the total stellar mass at $z=0$, while $55 \%$ was formed at $1<z<2$. Note that the peak of SF is actually at earlier times, $z \approx 3.5$, and that it decreases slowly to smaller $z$ in accordance with recent results from Cowie, Songaila and Barger (1999).

It has to be mentioned that numerical resolution affects SF more strongly at higher $z$ that at lower ones, and, that the inclusion of feedback mechanisms could have a non-negligible impact of the $\rho_{\mathrm{SFR}}$. However, current numerical models that include SN feedback are still limited by numerical and theoretical problems.

To sum up, the normalization of the power spectrum, the star formation efficiency and the minimum $T_{*}$ adopted in the SF model, affect the global SFR history of the simulated volumes and consequently, the SFR of each individual galaxy-like object. The value of $T_{*}$ is determined, in part by the available cooling functions, and indirectly, by numerical resolution of the gas component. The normalization parameter $\sigma_{8}$ is now defined within a narrow range depending on the adopted cosmology. However, many questions remain to be answered about the bias and its dependence on scale and red-shift. The value of $c$ is the actual free parameter of the SF model. In the following sections, we use these experiments (S.1, S.2 and S.3) to analyze the SF process within each galaxy-like object in relation to their merger histories, and assess how these parameters affect the individual SF histories. 


\subsection{Star Formation History and Mergers}

In this paper, we restrict the analysis to typical field galactic-like objects (GLOs). At $z=0$, GLOs are identified at their virial radius i.e., the radius for which the density contrast is estimated to be $\delta \rho / \rho \approx 200$ (White \& Frenk). We reject those GLOs with a comparable companion within two virial radii at $z=0$. In this way, we avoid complications due to tidal fields originated by the presence of several companions and/or the underlying over-density, focusing on the effects produced by the assembly of each individual object through hierarchical growth. In particular, in S.3 some objects have been discarded since they belong to groups. Each GLO is composed of a dark matter halo and a baryonic component in the form of gas and/or stars. We will only analyze in detail objects resolved with more than 250 baryonic particles within their virial radius at $z=0$. Table 1 gives the total dark matter $\left(\mathrm{N}_{\text {dark }}\right)$ and baryonic $\left(\mathrm{N}_{\mathrm{bar}}\right)$ particles within the virial radius for each GLO at $z=0$. The SF algorithm used is very effective at forming stars at the dense cores of the galactic-like objects, so it is easy to isolate star particle clumps.

We have named GLOs in S.1 and S.2 using the same label code as the one chosen in Tissera \& Domínguez-Tenreiro (1998) to identify halos. Simulations S.1 and S.2 correspond to their simulations I.2 and I.3. The main baryonic clumps in GLOs in S.1 that resemble a disk-like structure (DLO) have been studied from a dynamical point of view by Domínguez-Tenreiro et al. (1998). GLOs 3, 4, 5 and 6 in Table 1 host DLOs 1, 2, 4 and 3 in their Table 1, respectively.

We then follow back the evolution of the matter inside their virial radius as a function of the look-back time for the available outputs of the simulations. We construct the merger trees of each GLO identified at $z=0$ in the three simulations by recursively tracing back in time the objects which contain particles that end up in the final GLO. In this way, we individualize the progenitors and the satellites with which they merged at all outputs of the simulations (every 100 time-steps for S.1 and S.2, and 20 time-steps for S.3). All objects, progenitors and satellites, are identified at their virial radius at the corresponding $z$. The set of objects identified in this way gives a complete record of the merger history of each GLO. We will assume that the progenitor clump or parent galaxy of a GLO at $z=0$ is the most massive object identified at $z>3$ from 
its merger tree. A merger will be defined as the complete process since the time two baryonic objects are identified to share the same dark matter halo for the first time to their actual fusion. We will not distinguish between major and minor mergers. Instead, a merger will be counted each time the progenitor fusions with a satellite with more than $10 \%$ of its virial mass at that time of the merger. Otherwise, (i.e. less than $10 \%$ ) it will be considered accretion or infall. We can, then, follow the evolution with look-back time of each dark matter halo and its baryonic main clump. Some small satellites may have been formed and accreted between outputs. In this case, they would be missed by our analysis. However, this situation would happened only for very small objects that can be, anyway, counted as accretion. Note that we keep track of evolution of all smaller substructures that merge with the progenitor, but we do not look at them in detail as only their virial mass and gas content at the time of the merger are required for this analysis.

We estimate the star formation history of each GLO by reckoning the stellar mass formed in its progenitor objects at each $z$ and then, smoothing these distributions over time. The reason for adopting this procedure is that the SF model used in these simulations transforms a gas particle into a star one at once, after a time delay $\delta t$ over which the gas is supposed to be transformed into stars as explained in Section 2. A typical value for this time delay is $\langle\delta t>\approx 20 \Delta t$, where $\Delta t$ is the integration time-step of the simulations Then the $S F R$ have been smoothed by binning these distributions in time-bins of 20 points centered at the formation time of each star particle and averaging the stellar mass formed within each time-bin.

In Figure 2 we show, as an example, the star formation history from $z=1$ of the galactic objects 1, 2, 3 and 4 in simulation S.1. We have plotted the $\left\langle S F R>\left(\mathrm{M}_{\odot} / \mathrm{yr}\right)\right.$ in the progenitor object versus look-back time $\left(\tau(z)=1 .-(1+z)^{-2 / 3}\right.$ for $\left.\Omega=1\right)$. The times at which the satellite enters the virial radius of the progenitor has been indicated with an arrow pointing up, while the actual fusion of the baryonic cores has been indicated with an arrow pointing down. These are all the merger events in which the GLOs are involved in the range depicted in the figure. As can be seen in Figure 2, in all cases there is an increase of SF related to a merger with a satellite of more than $10 \%$ the progenitor mass. This situation is common to all GLOs in the other three 
simulations. From this figure it can be also seen that when a satellite enters the virial region of the progenitor, there is a delay in the fusion of the gaseous cores (Navarro, Frenk \& White 1995). During this interval, the objects orbit around each other and are under the effects of strong tidal fields. According to the analysis of some authors (e.g., Hernquist 1989a; Hernquist 1989b; Barnes 1988; Domínguez-Tenreiro et al. 1998), the interactions and fusions with satellites may supply gas to the central region of the parent galaxy fueling a burst of star formation.

Recall that among the different processes that can affect the SF history of the GLOs in hierarchical clustering scenarios (such as the assembly of the main object at high $z$, the merger of the progenitor with other clumps, gas compression as it cools and collapses inside dark matter halos during the quiescent phases of the assembly of the GLOs, and the interactions with neighboring structures that do not end up in actual fusions), in this work, we refer only to mergers (as defined in the Introduction). Consequently, we only study those well-defined peaks that are located within merger events (i.e., those within arrows in Figure 2). So we have not analyzed the first SF peaks that can be related with the assembly of the progenitor object (i.e., in Figure 2 peaks at $\tau(z) \approx 0.32$ in GLO 1 and 4 ). These peaks occur at a $z$ where the virial mass of the progenitor is always less than $\leq 20 \%$ of the final GLO virial mass, and they could be strongly affected by numerical resolution.

During the merger process (i.e. from the time the satellite enters the virial radius of the progenitor until one single baryonic clump forms) the progenitor and its satellite continue accreting gas. In our simulations, this fraction is important since, in same cases, it equals the amount of new stars formed. For example, GLO 2 in S1 transformed $30 \%$ of its original gas into stars during a merger with an object with a mass of $40 \%$ the progenitor mass. During that process, the amount of gas accreted was $29 \%$ of the gas mass of the final objects, and the ratio between the new stars and the old ones was 0.82 . The same object in S.2 transformed $83 \%$ of its initial total gas into stars, accreted $30 \%$ of the remnant gas and the burst resulted in a $25 \%$ increase of stellar mass. 


\subsection{Star Formation Peaks}

Because the star formation algorithm used in this work, in practice, transforms at once (after satisfying all the requirements mentioned in Section 2) a gas particle into a star one, the overall star formation history of a galaxy-like object is discrete and quite noisy. Although it is clear when there is a peak in the star formation history, this noise makes it difficult to isolate the stars formed in a single burst, and consequently, to classify the strength of the peak of new stars. In order to do so more rigorously, we took the following steps. We estimate the overall minimum star formation rate in a GLO, $\delta_{\min }$, at any red-shift. We, then, subtract a factor $f$ of this minimum from the total star formation rate history, so the peaks are clearly identified as the values with a signal larger than a threshold, $\sigma_{\min }=f \times \delta_{\min }$. We tried different values of $f$, choosing $f=3$ since this is the minimum one that allows us to individualize peaks in all GLOs in all simulations. Values below $\sigma_{\min }$ are considered 'ambient star formation rate' (ASFR). This ASFR can be explained as being driven by the increase of cold dense gas as the result of the cooling and collapse of baryons on to the potential well of the halo. We estimate that the ASFRs take values of $\leq 3 \mathrm{M}_{\odot} \mathrm{yr}^{-1}$. The mean values for $\left\langle\delta_{\min }>\right.$ are $0.90,1.45,0.89 \mathrm{M}_{\odot} / \mathrm{yr}$ for S.1, S.2 and S.3, respectively. The larger mean value measured for S.2 reflects the fact that the rate of star formation is always higher in this simulations (since $c$ is higher) than in the other ones. In Figure 2, the horizontal solid lines represent $\sigma_{\min }$ for each GLO. From this figure we see that the total star formation rate histories are composed of this approximately constant ambient star formation rate over which stellar bursts are superposed. 
Table 1. Main characteristics of star bursts.

\begin{tabular}{|c|c|c|c|c|c|c|c|c|}
\hline S & $G L O$ & $\mathrm{~N}_{\text {dark }}$ & $\mathrm{N}_{\text {bar }}$ & $\sigma_{\text {star }}$ & $M_{\text {burst }}$ & $\tau_{\text {burst }}$ & $M_{\text {sat }} / M_{\text {pro }}$ & $M_{\text {star }} / M_{\mathrm{bar}}$ \\
\hline \multirow[t]{17}{*}{ S.1 } & 1 & 9181 & 1335 & 18.75 & 0.55 & 4.36 & 0.16 & 0.12 \\
\hline & & & & 33.01 & 4.06 & 19.53 & 0.23 & 0.10 \\
\hline & & & & 15.47 & 1.12 & 8.61 & D & \\
\hline & 2 & 7310 & 1059 & 24.76 & 0.96 & 3.53 & 1.08 & 0.14 \\
\hline & & & & 16.52 & 0.10 & 1.99 & $\mathrm{D}$ & \\
\hline & & & & 46.43 & 4.63 & 17.60 & 0.39 & 0.26 \\
\hline & & & & 12.38 & 0.26 & 5.76 & $\mathrm{D}$ & \\
\hline & & & & 17.53 & 1.25 & 10.11 & 0.11 & 0.28 \\
\hline & 3 & 6317 & 917 & 13.41 & 0.91 & 11.50 & 0.40 & 0.22 \\
\hline & & & & 28.89 & 2.18 & 11.21 & $\mathrm{D}$ & \\
\hline & 4 & 7115 & 908 & 29.92 & 1.44 & 13.50 & 0.16 & 0.24 \\
\hline & & & & 12.38 & 0.65 & 10.39 & 0.16 & 0.27 \\
\hline & 5 & 5215 & 739 & 8.25 & 0.49 & 2.48 & 0.43 & 0.27 \\
\hline & & & & 19.60 & 0.89 & 12.4 & D & \\
\hline & & & & 22.70 & 0.91 & 5.14 & 0.22 & \\
\hline & 6 & 6184 & 798 & 16.50 & 1.71 & 11.50 & 0.40 & 0.15 \\
\hline & & & & 17.53 & 1.09 & 8.11 & $\mathrm{D}$ & \\
\hline \multirow[t]{11}{*}{ S.2 } & 1 & 9199 & 1307 & 42.30 & 0.68 & 3.94 & 0.18 & 0.58 \\
\hline & & & & 43.33 & 2.76 & 13.40 & 0.22 & 0.61 \\
\hline & 2 & 7383 & 1071 & 52.62 & 1.56 & 8.86 & 1.06 & 0.51 \\
\hline & & & & 30.96 & 1.72 & 6.01 & D & \\
\hline & & & & 71.19 & 4.18 & 19.08 & 0.38 & 0.54 \\
\hline & & & & 66.93 & 0.36 & 8.13 & 0.11 & 0.59 \\
\hline & 3 & 6501 & 918 & 30.95 & 1.56 & 11.21 & 0.43 & 0.62 \\
\hline & 4 & 7144 & 921 & 39.21 & 1.87 & 7.47 & 0.10 & 0.66 \\
\hline & & & & 41.12 & 1.53 & 13.99 & 0.21 & 0.46 \\
\hline & 5 & 5294 & 744 & 40.24 & 2.11 & 15.03 & 0.51 & 0.78 \\
\hline & & & & 33.02 & 1.43 & 19.30 & D & \\
\hline
\end{tabular}


For each stellar burst, we estimate the value of a local maximum $\left(\sigma_{\text {star }}\right)$, its duration $\left(\tau_{\text {burst }}\right)$ and the total amount of stars $\left(M_{\text {burst }}\right)$ formed during this period of time 1 . These last two parameters $\left(M_{\text {burst }}\right.$ and $\left.\tau_{\text {burst }}\right)$ are sensitive to $f$. In order to carry out a consistent analysis, once $f$ is chosen, it is kept constant for all objects in all simulations. In general, peaks have $\sigma_{\text {star }}>3 \sigma_{\min }$.

As already discussed, we have restricted this study to those peaks that can be directly related to merger events, after the main objects or progenitors are already formed and better resolved. In Table 1 we summarize the principal parameters that characterize those star formation peaks, including the ratios between the virial masses of the progenitor, $M_{\text {pro }}$, and the satellite objects, $M_{\text {sat }}$, at the time of the merger (i.e., the time when the satellite enters the virial radius of the parent GLO) and between the stellar mass content, $M_{\text {star }}$, of the system and its total baryonic mass, $M_{\mathrm{bar}}$, at the same time. The ratio $M_{\mathrm{star}}^{z} / M_{\mathrm{star}}^{0}$ is the fraction of the total stellar mass of the progenitor GLO at $z=0$ that has actually been formed at the red-shift of each merger $\left(M_{\mathrm{star}}^{z}\right)$. GLO 6 in S.2 has not been included since it is not possible to clearly isolate the star formation peaks associated with the mergers due to the high level of noise in its SF history. During some merger events, two stellar peaks have been detected. They are denoted by a letter D in Table 1 and will be discussed in more detail in a separate paper (Tissera et al. 2000). Note that we study a total number of 25 different merger events. So, even if the GLOs whose evolutionary histories are analyzed are restricted to the more massive ones, we look at all mergers recorded in their merger trees. Hence, this is equivalent to have performed 25 different mergers using controlled toy-models, with the advantage that each one of these mergers has physical properties determined by the underlying cosmology and the astrophysical model, and occurs at different stages of evolution. Unfortunately, this sample is not large enough to study the possible dependence of the stellar burst characteristics with the red-shift.

\footnotetext{
${ }^{3}$ Peaks have to be determined by more than three points higher than $\sigma_{\min }$ in order to be classified as a stellar burst; and their durations, $\tau_{\text {burst }}$, are estimated as the period of time comprised between the first point to surpass this threshold and the last point which satisfies this condition.
} 
Table 1-Continued

\begin{tabular}{|c|c|c|c|c|c|c|c|c|}
\hline $\mathrm{S}$ & $G L O$ & $\mathrm{~N}_{\text {dark }}$ & $\mathrm{N}_{\text {bar }}$ & $\sigma_{\text {star }}$ & $M_{\text {burst }}$ & $\tau_{\text {burst }}$ & $M_{\text {sat }} / M_{\text {pro }}$ & $M_{\mathrm{star}} / M_{\mathrm{bar}}$ \\
\hline \multirow[t]{8}{*}{ S. 3} & 1 & 3688 & 550 & 23.73 & 0.57 & 0.81 & 0.31 & 0.10 \\
\hline & & & & 5.16 & 0.05 & 1.23 & $\mathrm{D}$ & \\
\hline & 2 & 5665 & 1056 & 8.25 & 0.60 & 14.25 & 0.58 & 0.15 \\
\hline & & & & 5.16 & 0.44 & 13.38 & $\mathrm{D}$ & \\
\hline & 3 & 1168 & 272 & 22.69 & 0.43 & 2.89 & 0.35 & \\
\hline & & & & 6.19 & 0.18 & 3.12 & 0.10 & \\
\hline & 4 & 3302 & 567 & 18.57 & 0.47 & 2.70 & 0.24 & 0.26 \\
\hline & & & & 10.32 & 0.36 & 2.76 & D & \\
\hline
\end{tabular}

Note. - Units: $\left[\sigma_{\text {star }}\right]=\mathrm{M}_{\odot} / \mathrm{yr} ;\left[M_{\text {burst }}\right]=10^{10} \mathrm{M}_{\odot} ;\left[\tau_{\text {burst }}\right]=10^{8} \mathrm{yr}$ 
Let us now try to investigate if the parameters that characterize the stellar bursts are consistent with observations of galaxies undergoing strong stellar activity at different red-shifts and how they change with the model parameters $\left(\sigma_{8}, c, T_{*}\right)$. As can be seen from Table 1 , the values of $\tau_{\text {burst }}, M_{\text {burst }}$ and $\sigma_{\text {star vary among simulations and are different for S.1 and S.2 versions }}$ of the same merger event. In Table 2 are shown the mean values $\left\langle\sigma_{\text {star }}\right\rangle,\left\langle\tau_{\text {burst }}\right\rangle$, and $<M_{\text {burst }}>$ in units of $\mathrm{M}_{\odot} / \mathrm{yr}, 10^{8} \mathrm{yr}$ and $10^{10} \mathrm{M}_{\odot}$, respectively. As expected, the higher values are measured for peaks in S.2 since, as discussed in Section 2, the gas is transformed into stars more efficiently in this simulations than in S.1 and S.3. Recall that the only difference between S.1 and S.2 is the $c$ parameter, and, that S.1 and S.3 have different bias parameter and critical temperature, $T_{*}$, but equal $c$ value. In S.3, at lower $z$, the stellar peaks are less important when compared to those in S.1, because a larger fraction of gas has been consumed at higher $z$ (so the gas density is lower at later times) and also because of the more restrictive temperature criterium $T_{*}$ used. At those low $z$, GLOs in S.1 are more gas-rich and can produce stronger stellar bursts. For simulation S.3, we see that the mean stellar mass, $<M_{\text {burst }}>=3.90 \times 10^{9} \mathrm{M}_{\odot}$, and the starburst time-scale, $<\tau_{\text {burst }}>=5.14 \times 10^{8} \mathrm{yr}$, are consistent with observed values inferred for starburst galaxies and high $z$ objects undergoing important star formation activity (see Sawicki \& Yee 1997; Kennicutt 1998 and references therein). Also note that these values depend on $f$. Had we chosen a higher threshold $\left(\sigma_{\min }\right), \tau_{\text {burst }}$ and $M_{\text {burst }}$ would have been smaller in all cases. Note also that the simulated bursts occur at different $z$, so a direct comparison with observed objects at high $z$ is complex since, for example, the effects of dust reddening are still uncertain and lead to underestimations of the SFRs. And, on the other hand, a comparison with only local starburst galaxies could be misleading, since the properties of the galactic objects change with $z$ and so could do their SF histories (e.g., Guzmán et al. 1997; Driver et al. 1998; Flores et al. 1998).

The next question is in relation to the factors triggering these stellar bursts and if cause-effect relations can be isolated. We estimate the ratio between the virial mass of the satellite $\left(M_{\text {sat }}\right)$ that is falling in, and the virial mass of the progenitor $\left(M_{\text {pro }}\right)$ just before the satellite enters the virial radius of the parent galaxy. We plot $\sigma_{\text {star }}$ vs. $M_{\text {sat }} / M_{\text {pro }}$, including the double peaks (see Section 3.3) in Figure 3a. The response is different depending on the simulation. In simulation 
$\mathrm{S} .2, \sigma_{\text {star }}$ values are higher than those in S.1 although the GLOs are numerically identical have the same merger trees in both simulations. This difference is originated in the different star formation efficiency as already discussed: it is easier to transform a gas particle into a star one in S.2 than in S.1. The same argument is valid for S.3 which has the lower values for roughly the same $M_{\text {sat }} / M_{\text {pro }}$ ratios. Note that events with approximately equal $M_{\text {sat }} / M_{\text {pro }}$ ratios produce different $\sigma_{\text {star }}$ in the same simulation.

An interesting fact observed from this figure is that, even a merger with a low mass satellite, $M_{\text {sat }} / M_{\text {pro }} \approx 0.10$, correlates with an increase of the star formation rate in all simulations, with $\sigma_{\text {star }}$ values similar to those corresponding to higher $M_{\text {sat }} / M_{\text {pro }}$. This result is in agreement with Mihos \& Hernquist 1996 who studied in detail the accretion of low mass satellites and found that even a merger with an object with a mass of $10 \%$ the parent galaxy mass, can produce an inflow of gas to the center of the main object fueling a starburst. This result may suggest that a comparable companion is not a necessary condition for triggering star formation, but a smaller one may produce significant effects.

The availability of gas in condition of forming stars is one necessary condition to trigger a stellar burst. Hence, it would be expected a correlation between the gas content of the system and the strength of the stellar peaks. In Figure $3 \mathrm{~b}$, we plot $\sigma_{\text {star }}$ vs. $M_{\text {gas }} / M_{\text {bar }}$ where $M_{\text {gas }}$ is the total gas mass of the satellite and its progenitor within $r_{200}$, before the merger, and their total baryonic

Table 2. Mean values for the parameters of the stellar bursts.

\begin{tabular}{lrrr}
\hline \hline $\mathrm{S}$ & $\left\langle\sigma_{\text {star }}\right\rangle$ & $\left\langle\tau_{\text {burst }}\right\rangle$ & $<M_{\text {burst }}>$ \\
\hline & & & \\
1 & 20.79 & 9.28 & 1.36 \\
2 & 40.02 & 11.49 & 1.79 \\
3 & 12.51 & 5.14 & 0.39 \\
\hline
\end{tabular}


mass $M_{\mathrm{bar}}$ (gaseous and stellar masses of the satellite and the progenitor together at $r_{200}$ ) at the same time, for the objects in S.1, S.2 and S.3. It can be seen that there is no correlation. Note also that not always the more gas-rich objects have the larger $\sigma_{\text {star }}$ or that equally gas-rich objects have different $\sigma_{\text {star }}$, even in the same simulations, that is, with the same SF parameters. However, the absolute value of the bursts depends on $c$ : S.2 has higher $\sigma_{\text {star }}$ values even though the objects are more gas-poor than their counterpart in S.1. Note also that within the same simulation, GLOs have very similar gas abundances. Hence the fact that equal massive mergers produce different $\sigma_{\text {star }}$ in the same simulation cannot be directly related to a difference in gas richness of the objects involved.

Given the durations of the bursts, $\tau_{\text {burst }}$, and the total stellar mass formed during that period, $M_{\text {burst }}$, it is possible to estimate an overall star formation rate $\left.<S F R\right\rangle_{\text {burst }}=M_{\text {burst }} / \tau_{\text {burst }}$ associated to the burst. We found values ranging from $50 \mathrm{M}_{\odot} / \mathrm{yr}$ to $2 \mathrm{M}_{\odot} / \mathrm{yr}$ depending on the simulation. For illustration purposes, we plot them against the the ratio $M_{\text {sat }} / M_{\text {pro }}$ (Figure $3 \mathrm{c}$ ). Again, we found no correlation signal. Since $<S F R>_{\text {burst }}$ depends not only on the amount of stellar mass formed, but also on the duration of the burst, this lack of correlation is not surprising. The duration of the bursts, $\tau_{\text {burst }}$, can be determined by different parameters such as orbital orientation, internal structure, star formation efficiency, in a complex way. From this figure we can also observe that a merger with a satellite of 0.10 or 0.40 the mass of the parent object may produce the same average $\langle S F R\rangle_{\text {burst }}$ within the same simulation. This fact supports the idea that it is not only the masses of each component in each halo that matters, but other factors could also be relevant, such as the dynamical characteristics of the encounter and the structural properties of the baryonic clumps that merge. We have also searched for possible correlations between both, $\tau_{\text {burst }}$ and $M_{\text {burst }}$, with $M_{\text {sat }} / M_{\text {pro }}$ and $M_{\text {gas }} / M_{\text {bar }}$. No signal was detected implying that the total stellar mass and the burst duration are not simple functions of only the relative masses of the merging objects or their gas richness. However, they do depend on $c$ as already pointed out (see Table 1).

These results prompt us to differentiate between minor and major mergers in order to 
ascertain if a hidden effect could be disentangled. In Figure 4 a we plot again $\sigma_{\text {star }}$ vs. $M_{\text {gas }} / M_{\text {bar }}$ but, in this case, filled symbols represent major mergers, while open ones, minors events (circles, triangles and pentagon for S.1, S.2 and S.3, respectively). The limit between major and minor mergers has been set at $M_{\text {sat }} / M_{\text {pro }}=0.35$ (Baugh et al. 1996). We include single bursts and the first components of double ones (secondary components are formed with the remanent gas after the first one, so actually they depend on the properties of the first component). We can see from this figure that a minor merger can trigger a burst as strong as a major one, even if the systems is less gas-rich, and that a major merger, in some cases, does not trigger a strong burst even in gas-rich collisions.

To deepen into the burst process, we compare the amount of stars formed in a given stellar burst, $M_{\text {burst }}$, with the amount of gas available in the system to form stars, $M_{\text {gas }}$. In Figure $4 \mathrm{~b}$ we do not see any clear correlation, that is, the amount of gas available at the beginning of the merger that is actually transformed into stars does not mainly depend on $M_{\text {gas }}$. In most cases, it is smaller than the amount of gas available in the system, so the gas mass is not actually completely exhausted during a burst. This can also be seen in the SFR histories, from where we observe that the SF continues after a burst, although at a lower rate.

An estimate of the efficiency of the star formation process in each burst can be defined as the fraction of available gas in the system, at the time the satellite enters the virial radius of the progenitor, that was actually converted into stars, $M_{\text {burst }} / M_{\text {gas }}$. We plot this burst efficiency ratio versus $M_{\text {gas }} / M_{\text {bar }}$ (Figure 5a) and $M_{\text {sat }} / M_{\text {pro }}$ (Figure 5b) for the single bursts and for the first peaks of double bursts. As can be seen from these figures, there is no correlation between the burst efficiency and the gas abundance of the GLOs, implying that, in these GLOs, the burst efficiency is not determined by the gas abundance (neither is there a correlation of the efficiencies with $M_{\text {gas }}$, which suggest that they are doubtfully determined by numerical resolution). But a trend is present with $M_{\text {sat }} / M_{\text {pro }}$, suggesting that massive mergers can induce more efficient transformations of the gas into stars. The mean values of $M_{\text {burst }} / M_{\text {gas }}$ for stellar bursts associated with minor $\left(M_{\text {sat }} / M_{\text {pro }}<0.35\right)$ and major $\left(M_{\text {sat }} / M_{\text {pro }} \geq 0.35\right)$ mergers for S.1, S.2 and S.3 are 
$(0.24,0.35),(0.23,0.72)$ and $(0.10,0.19)$, respectively. We have averaged all peaks regardless of the red-shift at they have occurred.

This trend has been reported by Mihos \& Hernquist (1994, 1996) who used high-resolution models of pairs of merging galaxies, and successfully implemented by Somerville, Primack \& Faber (1998) in a semi-analytic model. We found the same relation for objects formed in a cosmological context and for a range of merger parameters that naturally arises in coherence with the stage of evolution of the GLOs. However, our efficiencies are not as high as those claims by Somerville et al. (1998) and depend on the SF parameters.

All these results suggest that, at least, a third parameter is playing a role in the triggering of the bursts: it is not enough to have available gas in the system, independently of the relative mass of the colliding objects. The gas has to be violently compressed in short time-scales in order to induce a starburst, and in this case, mergers seem to be doing part of the work.

Concerning numerical resolution, the lack of correlation found between the burst characteristics and the gas abundance $\left(M_{\text {gas }} / M_{\text {bar }}\right)$ or the gas mass $\left(M_{\text {gas }}\right.$, that gives a rough idea of the gas resolution of the system at the time of the merger) strongly suggest that they are not determined by numerical resolution. A second fact that supports this point is that peaks in S.2 have higher $\sigma_{\text {star }}$ values than those of the same GLOs in S.1, even though the objects are more gas-poor than those in S.1.

Because the SF process depends directly on the gas density, its correct numerical description is crucial. As shown by Tissera \& Domínguez-Tenreiro (1998) and Domínguez-Tenreiro et al (1998), the gas density within the dark matter halos of massive systems in these simulations are rather well described. In this sense, an advantage of these simulations is that the mass of gas and dark particles are equal, implying that the dark matter is resolved with a factor of about 10 more particles than the gas. This fact assures that two-body effects are unimportant and that the dark matter profiles are well represented in the central regions. An adequate resolution of the dark matter profiles strongly helps the gas to cool and collapse inside the central regions following a correct density profile (Steinmetz \& White 1997). This implies that the SF process, that depends 
on the gas density, can be also adequately followed.

\section{Stellar Population and Color Distributions}

As already discussed in Section 3, the star formation history of each individual galactic object can be followed with look-back time. This information can be combined with stellar population synthesis models to estimate the luminosities and colors of galactic objects throughout their evolutionary history (Tissera et al. 1997).

We use the models of Bruzual \& Charlot (1993) to calculate the luminosity of a particle as a function of wavelength $\lambda$ and $z$. We assume a Miller-Scalo initial mass function with a lower mass cutoff of $0.1 M_{\odot}$ and an upper mass cutoff of $125 M_{\odot}$, and a burst of 20 time-steps duration $\left(2.8 \times 10^{8}\right.$ yr for S.1 and S.2, and $2.4 \times 10^{8}$ for S.3 $)$ for each particle. Then, we sum up the luminosities of the particles belonging to an object and, from the total luminosities, we estimate their colors and magnitudes at different wavelengths. No supernova energy injection or metallicity enrichment have been included. We have neither included reddening effects so some comparison with observed data may result to be rather crude and unfair to simulated colors.

In Figure 6 we plot $U-B$ vs $B-V$ at $z=0$ for the simulations analyzed: S.1, S.2 and S.3. We also include the observational data obtained from the RC3 catalogue (de Vaucouleurs et al. 1991) which has UBV photometry, Hubble types and measured red-shifts for $\sim 10000$ galaxies. We plot $U-B, B-V$ locus for both early and late-type galaxies with $z \leq 0.05$ in RC3. We see that most of the simulated GLOs are bluer than the galaxies although the maximum departure from the observations is at most of $\approx 0.3$ magnitudes. This is clearly due to fact that the star formation rate histories of the simulations produce higher rates than observed at $z<0.05$. But in spite of this fact, a better agreement between simulated and observed colors is not impossible and it only requires a lower star formation rate at $z \approx 0$ which can be accomplished, for example, by using a lower $c$ value or including SN feedback. It is also possible that, because at high $z$, GLOs are not very well resolved in these simulations, the gas is inefficiently consumed into stars leading to more 
gas-rich GLOs at lower $z$. On the other hand, higher resolution experiments without a correct feedback implementation could lead to a very effective SF process at high $z$ producing GLOs with lower SFR and redder colors at $z=0$ (e.g., Steinmetz \& Navarro 1999). In our simulations, low resolution at very high $z$ acts as a feedback effect.

Note that the SFR histories among the simulations are quite different (because of the different SF parameters used), however the distributions of colors at $z=0$ on this color-color diagram are very similar. Hence these colors are very insensitive to the star formation history of the GLOs and are not a very safe way of adjusting SF parameters in semi-analytic or numerical models.

In Figure 7 we plot $B-V$ vs $z$ for four galactic-like objects in simulations S.1 and S.2. Although the number of outputs available is small, it is still possible to follow the color evolution of the progenitor. It can be clearly seen that the color path is not smooth. An object can move from blue to red and back to blue colors depending on their history. The change in colors in these models can be as large as half a magnitude. In particular, each change in direction in the color-color diagrams correlates with a peak of star formation as expected. The strength in the change depends on the relative number of the new to the old stars. In this figure we have plotted the tracks of objects in S.1 and S.2 with the aim at comparing the influence of the SF efficiency. As can be seen, although the general behavior is similar, the detailed evolution is different. The $B-V$ tracks of GLOs in S.2 are displaced in time with respect to those in S.1. Since the only difference between these simulations is their star formation efficiency parameters, the color evolution of the objects could be affected by its choice.

An even clearer approach for analyzing the evolution of the stellar population and its relation with mergers is to look at $B-I$ vs $z$. In Figure 8 we plot it for the same four objects in S.1 shown in Figure 7 (solid lines for a Miller-Scalo IMF). This figure shows clearly when there is a burst of star formation and its correlation with mergers (the red-shift at which the satellite enters the virial radius of the progenitor has been indicated with an arrow pointing up, while the actual fusion of the baryonic cores has been indicated with an arrow pointing down). In some cases, because of the small number of outputs, we could have missed the peak and just see the remnants. 
Note that this problem is not present in the star formation history since it is saved completely until $z=0$. Again, the changes in color depend on the particular characteristic of the mergers and the proportion of old to new stellar populations. We also estimate colors using a Salpeter IMF with the same lower and upper cutoffs (dashed lines). As can be seen the differences are very small, thou the colors calculated using Salpeter IMF tend to be redder. Unfortunately, the smaller stellar mass allowed by Bruzual \& Charlot's models is $0.1 M_{\odot}$, so we could not evaluate the effects of assuming a lower mass cutoff such as $0.01 M_{\odot}$. In Figure 9, we plot $B-I$ vs $z$ for the 4 GLOs shown in Figure 7 and 8 (simulations S.1 and S.2) and observed values from the LDSS2 (Ellis et al. 1996) and LRIS (Guzmán et al. 1997). Despite the $\rho_{\text {SFR }}$ are very different between these simulations, their evolutionary color tracks are in general agreement with observations.

Obviously not all star bursts are triggered by mergers, but, since they are common events in a hierarchical scenario, their possible influence on the evolution of colors cannot be ignored. This picture deduced from the star formation histories and color evolutionary tracks resembles the 'Christmas Tree' model discussed by Lowenthal et al. (1997) in which individual star forming blobs come and go. According to our models, the star formation in this blobs would be the result of two contributions: one due to an approximately constant ambiance star formation and a certain number of SF peaks. The aggregation of substructure according to a hierarchical scheme would be one possible mechanism of SF triggering. The parent galaxy will evolve by undergoing a number of mergers which may trigger starbursts depending on the particular characteristic of the encounters, and the physical properties of the objects involved. Hence during violent phases colors would became bluer to change again to redder ones as a quiescent period takes place. Moreover, because of the way the structure forms in a hierarchical scenario, the higher the $z$, the higher the probability that massive objects would be observed to be undergoing an important star formation activity period (Guzmán et al. 1997) since the rate of mergers increase with $z$, GLOs are gas richer and the gas is denser.

This technique that combines hydro-dynamical simulations and synthesis evolutionary models has proved to be potentially powerful to study the formation of galaxies as a function of $z$. 
Nevertheless, more complex models will be needed in order to mitigate numerical resolution problems, and to allow us to numerically follow the evolution of galactic structure of different masses with look-back time.

\section{Summary}

We have analyzed the history of star formation in galactic objects simulated within the framework of a clustering hierarchical model. Our aim was to use a set of three cosmological simulations to study the possible interplay between hierarchical aggregation and star formation. We found that, if the structure in the Universe is well represented by a hierarchical clustering model, then our results suggest that the process of aggregation of substructure could be one of the mechanisms that triggers star formation in galactic systems. In this work, SN effects have not been included. It is expected that they will contribute to set a self-regulated SF. However, their actual impact on galaxy scales remains to be clearly stablished.

Our conclusions can be summarized as follows:

I. The star formation rates as a function of $z$ of our simulated GLOs have two components: one approximately constant $\left(\mathrm{ASFR}<3 \mathrm{M}_{\odot} / \mathrm{yr}\right)$, and a series of stellar bursts superposed. We found that the aggregation of substructures by the progenitor objects correlates with the presence of stellar bursts. These bursts last $10^{8}-10^{9} \mathrm{yr}$ and produce stellar masses of $10^{9}-10^{10} \mathrm{M}_{\odot}$. For S.3, these parameters are consistent with observations of galaxies undergoing strong SF activity at different $z$. For S.1 and S.2 the values are higher as could be predicted from the global $\rho_{\mathrm{SFR}}$.

II. No correlation between the strength of the stellar peaks, its duration and stellar mass formed, on one hand, and the ratios $M_{\mathrm{sat}} / M_{\mathrm{pro}}$ and $M_{\mathrm{gas}} / M_{\mathrm{bar}}$, on the other hand, was found. This fact implies that they do not determine the characteristics of the burst by their own. And that the strength of a stellar peak can not be predicted only from the gas richness or the size of the colliding satellite. Mergers with equally massive objects produce different effects in the same simulation. When major and minor mergers are distinguished it can be seen that both of them 
can generate different stellar maxima regardless of their mass content. The strength of the star formation peaks, however, do depend on the SF efficiency parameter used in the models.

III. We found a trend for massive mergers to be more efficient at inducing a transformation of the gas available in the system into stars.

IV. In agreement with Hernquist (1989b), we find that a merger with a satellite of even $10 \%$ of the progenitor mass can be correlated with a stellar burst independently of the value of SF efficiency used. This result would imply that, when searching for a companion as the triggering factor of strong star formation activity in a galaxy, not only similar galaxies should be checked out but, also smaller ones (Donzelli \& Pastoriza 1997).

V. The color tracks of GLOs are not smooth, but go from bluer to redder in the quiescent phases of evolution, and vice-versa in the violent phases corresponding to mergers. The amount by which colors change on the latter depend mainly on the star formation history of each GLO. But color distributions at $z=0$ are quite insensible to their particular star formation histories.

The author is grateful to Prof. Diego G. Lambas, Rachel Somerville and the anonymous referee of this paper for stimulating discussions and comments. We thank Francois Hammer for providing useful information. P.B.Tissera thanks Imperial College the University of Oxford and the Centro de Computación Científica (Universidad Autónoma de Madrid) and the University of Oxford for providing the computational support for this work and for their hospitality. This work was partially supported by DGES (Spain), through grant PB96-0029 and Consejo Nacional de Ciencia y Tecnologia (Conicet, Argentina). 


\section{REFERENCES}

Barnes, J., 1988, ApJ, 331, 699

Barnes, J., Hernquist, 1996, ApJ, 471, 115

Barnes, J., Hernquist, 1991, ApJ, 370, L65

Baugh, C. M., Cole, S. \& Frenk, C. S., 1996, MNRAS, 283, 1361

Bouwens, R., Broadhurst, T., Silk, J., 1997 (astrohp/9710291)

Brinchmann et al 1998, ApJ, 499, 112

Bruzual, G. \& Charlot, S., 1993, ApJ, 405, 538

Burles, S, Tytler, D., 1998a, ApJ, 499, 699

Burles, S, Tytler, D., 1998b, ApJ, 507, 732

Cen, R., Phelps, S., Miranda-Escudé, J., Ostriker, J. P., 1998, ApJ, 496, 577

Clements, D., Sutherland, W.J., McMahon, R.G., Saunders, W., 1996, MN, 279, 477.

Connolly et al. 1997, ApJ, 486, 11L

Cowie, L. L., Songaila, A., Hu, E. M., Cohen J. G., 1996, ApJ, 112, 839

Cowie, L. L., Songaila, A. \& Barger, A., 1999 astro-hp/9904345)

Dalgarno, A., McCray, R.A., 1972, ARA\&A, 10, 375

de Vaucouleurs, G., de Vaucouleurs, A., Corwin, H.G., Buta, R.J., Paturel, G. \& Fouque, P., 1991, Springer-Verlag

Domínguez-Tenreiro, R., Tissera, P., Sáiz, A., 1998, ApJ, 508, L123

Donzelli, C. J., Pastoriza, M. G., 1997, ApJS, 111, 181 
Driver, S. P., Fernandez-Soto, A., Couch, W. J., Odewahn, S.C., Windhorst, R. A., Lanzetta, K., Yahil, K., 1998 (astro-ph/9802092)

Ellis, R. S., Colless, M., Broadhurst, T., Heyl, J., Glazebrook, K., 1996, MN, 280, 235

Ferrini, 1997. In press

Flores, H., Hammer, F., Thuan, T. X., Césarsky, C., et al. 1998, ApJ. In press.

Friedli, D., Benz, W., 1995, AA, 301, 649

Fukigita, M., Hogan, C. J., Peebles, P. J. E., Nature, 1996, 381, 489

Gallego, J., Zamorano, J., Arágon-Salamanca, A., Rego, M., 1995, ApJ, 455, L1

Gerritsen, J., 1997, PhD Thesis

Glazebrook, K., Ellis, R., Santiago, B., Griffiths, R., 1995, MNRAS, 275, L19

Guzmán, R., Gallego, J., Koo, D. C., Phillips, A. C., Lowenthal, J. D., Faber, S. M., Illingworth, G. D., Vogt, N. P., 1997, ApJ, 489, 559

Hernquist, L., 1989a, Nature, 340, 687

Hernquist, L., 1989b, Ann. NY Acad. Sci., 571, 190

Hernquist, L., Katz, N., Weinberg, D. H., Miranda-Escudé, J., 1996, ApJ, 457, L57

Hughes, D. et al. 1998, Nature, 394, 241

Kauffmann, G., White, S. D. M., Guiderdoni, B., 1993, MNRAS, 264, 201

Joseph, R.D. \& Wright, G.S., 1985, MNRAS, 214, 87.

Katz, N., 1992, ApJ, 391, 502

Katz, N., Hernquist, L., Weinberg, D. H., 1999, Highly Redshifted Radio Lines, ASP Conf. Series Vol. 156, Ed. by C. L. Carilli, S. J. E. Radfordm K. M. Menten, \& G. I. Langston, p.1 
Kennicutt, R. Jr., 1996, AAAS, 189, 6807

Kennicutt, R. Jr., 1998, astro-ph/9801188

Lacey, C., \& Silk, J., 1991, ApJ, 381, 14

Lawrence, A., Rowan-Robinson, M., Leech, K., Jones, D.H.P. \& Wall, J.V., 1989, MN, 240, 329.

Lilly, S. J., Tiesse, L., Hammer, F., Crampton, D., Le Fevre, O., 1995, ApJ, 441,18

Lilly, S., Le Fevre, O., Hammer, F. \& Crampton, D., 1996, ApJ, 460, 1.

Lilly, S. et al. 1997 astro-ph/9712061

Lowenthal, J. D., Koo, D. C., Guzman, R., Gallego, J., Phillips, A. c., Vogt, N. P., Illingworth, G. D., Gronwall, C., 1997, ApJ, 482, 673

Lucas, R. A., Borne, K. D., Bushouse, H., Colina, L., 1997, AAS, 190, 4305L

Madau, M. P., 1995, ApJ, 441, 18

Madau, M. P., Ferguson, H. C., Dickinson, M. E., Giavalisco, M., Steidel, C. C., Fruchter, A., 1996, MNRAS, 283, 1388

Madau, M. P., 1998 astro-ph/9801005

Metzler, C., Evrard, A., 1995, ApJ, 437,564

Mihos, J. C., Hernquist L., 1994, ApJ, 437, L47

Mihos, J. C., Hernquist, L., 1996, ApJ, 464, 641

Mobasher, B., Mazzei, P., 1998. Preprint

Navarro, J. F., White, S. D. M., 1993, MNRAS, 265, 271

Navarro, J. F., White, S. D. M., 1994, MNRAS, 267, 401 
Navarro, J. F., Frenk, C., White, S. D. M., 1995, MNRAS, 275, 56

Navarro, J. F., Steinmetz, M., 1997, ApJ, 478, 13

Pearce, F. R., Jenkins, A., Frenk, C. S., Thomas, P. A., Colberg, J. M., White, S. D. M., Couchmann, H. M. P., Peacock, J. A., Efstathiou, G., Nelson, A. H., 1999, ApJL accepted

Renzini, A., 1998 (astro-ph/9810304)

Sanders, D., Soifer, B.T., Elias, J.H., Madore, B.F., Matthews, K., Neugebauer, G. \& Scoville, N.Z., 1988, ApJ, 3255, 74

Sanders, D. B., Mirabel, F. I., 1996, AnRevAA, 34, 749

Sawicki, M., Yee, H. K., 1997 (astro-ph/9712216)

Silk, J., 1997, ApJ, in press.

Somerville, R., Primack, J., 1998 (astro-ph/9811001)

Somerville, R., Primack, J., Faber, S. M., 1998 astro-ph/9806228

Steidel, C. C., Hamilton, D., 1992, AJ, 104, 941

Steidel, C. C., Adelberger, K. L., Giavalisco, M., Dickinson, M., Pettini, M., 1998a, ApJ, submitted (astro-ph/9811399)

Steinmetz, M., White, S. D. M., 1997, MNRAS, 288, 545

Steinmetz, M., Navarro, J. F., 1999, ApJ, 513, 555

Theuns, T., Leonard, A., Efstathiou, G., Pearce, F. R., Thomas, P. A., 1998, MNRAS, 301, 478

Tissera, P. B., Lambas, D. G., Abadi M., G., 1997, MNRAS, 286, 384

Tissera, P. B., Domínguez-Tenreiro, R., 1998, MNRAS, 297, 177 
Tissera, P. B., Sáiz, A., Domínguez-Tenreiro, R.,1999, in preparation

White, S. D. M., Frenk, C., 1991, ApJ, 379, 52

Yepes, G., Kates, R., Khokhlov, A., Klypin, A., 1997, MNRAS, 284, 235 
Fig. 1.- Cosmic star formation rate density, $\rho_{\mathrm{SFR}}$, versus $z$ for simulations S.1 (dotted lines), S.2 (dashed lines) and S.3 (solid lines). We also include observational values reported by Gallego et al. (1995, asterisk), Madau et al. 1996 (filled triangles), Lilly et al. (1996, open squares), Connolly et al. (1997, ten arm stars), Sawicki et al. (1997, open stars), Hughes et al. (1998, filled square), Flores et al. (1998, crossed open squares) and Steidel et al. (1998, crossed open cricles).

Fig. 2.- Star formation rate history of four galactic-like objects analyzed in simulation S.1 as a function of look-back time. The horizontal solid line represents the ambient star formation rate $\sigma_{\min }$ for each object. The time at which the satellite enters the virial radius of the progenitor has been indicated with an arrow pointing up, while the actual fusion of the baryonic cores has been indicated with an arrow pointing down. Globally, the SFR of the GLOs can be described by the superposition of a constant star formation rate and a series of bursts.

Fig. 3.- The local maximun of star formation $\sigma_{\text {star versus the ratio between the virial masses }}$ of the satellite and the progenitor, $M_{\text {sat }} / M_{\text {pro }}$ (a) and the ratio between the gas content and the total baryonic mass of the colliding system, $M_{\mathrm{gas}} / M_{\mathrm{bar}}(\mathrm{b})$, and the average star formation rate $<S F R>_{\text {burst }}$ versus $M_{\text {sat }} / M_{\text {pro }}(\mathrm{c})$, for starbursts identified in galactic-like objects in simulations S.1 (open circles), S.2 (filled triangles), and S.3 (filled pentagons; for the sake of clarity, we have not included in figure c the data corresponding to the first burst of GLO 1 in S.3.: $M_{\text {sat }} / M_{\text {pro }}=0.31$ and $\left\langle S F R>_{\text {burst }}=70.37 \mathrm{M}_{\odot} / \mathrm{yr}\right.$.

Fig. 4.- a) $\sigma_{\text {star }}$ versus $M_{\text {gas }} / M_{\text {bar }}$ for GLOs in S.1 (circles), S.2 (triangles) and S.3 (pentagons). Solid symbols represent major mergers while open ones, minors. b) Stellar mass formed during a burst, $M_{\text {burst }}$, as a function of the total gas mass available in the system, $M_{\text {gas }}$ (symbol code as in Figure 3). Single bursts and first components of double ones have been included.

Fig. 5.- The fraction of gas converted into stars in a star formation burst, $M_{\text {burst }} / M_{\text {gas }}$, versus the gas fraction of the system $M_{\text {gas }} / M_{\text {bar }}$ (a) and the relative masses of the colliding objects $M_{\text {sat }} / M_{\text {pro }}$ (b), for single peaks and the first components of double ones. A trend is found for massive mergers to produce more efficient bursts. 
Fig. 6.- $U-B$ vs $B-V$ diagram for GLOs at $z=0$ in S.1 (open circles), S.2 (filled triangles) and S.3 (filled pentagons). We also plotted colors for early (five arm stars) and late (ten arm stars) types galaxies from the RC3 catalogue.

Fig. 7.- $B-V$ colors versus $z$ for four objects in simulations S.1 (open circles) and S.2 (filled triangles).

Fig. 8. $-B-I$ colors versus $z$ for the same four objects in simulations S.1 estimated by using two different IMF: Miller-Scalo (solid line) and Salpeter $(\mathrm{x}=1.5$; dotted line).

Fig. 9.- Comparison between the $B-I$ tracks of GLOs in S.1 (dashed lines) and S.2 (solid lines) with observation data: LRIS (filled circles) and LDSS2 (open circles). 


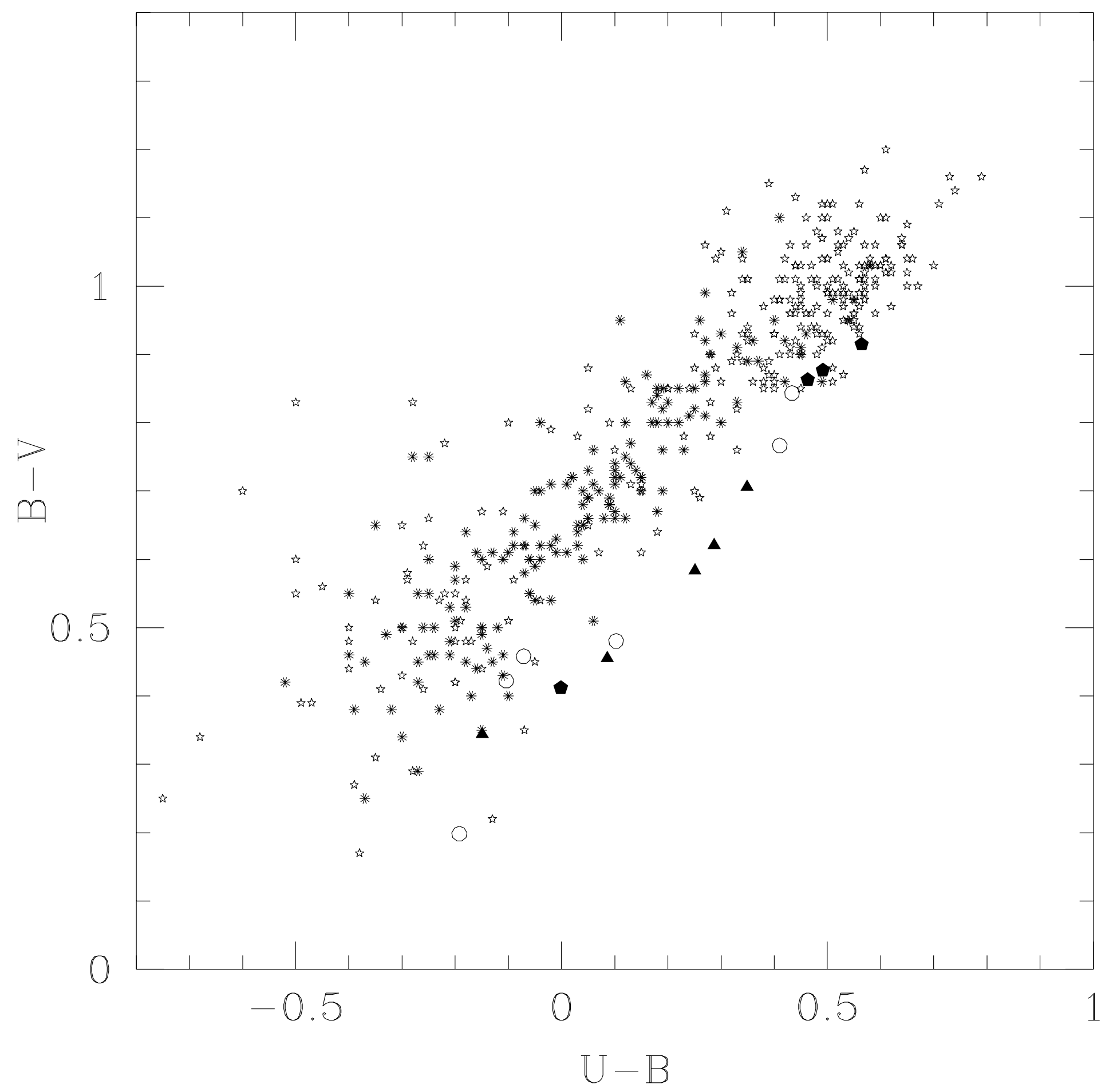




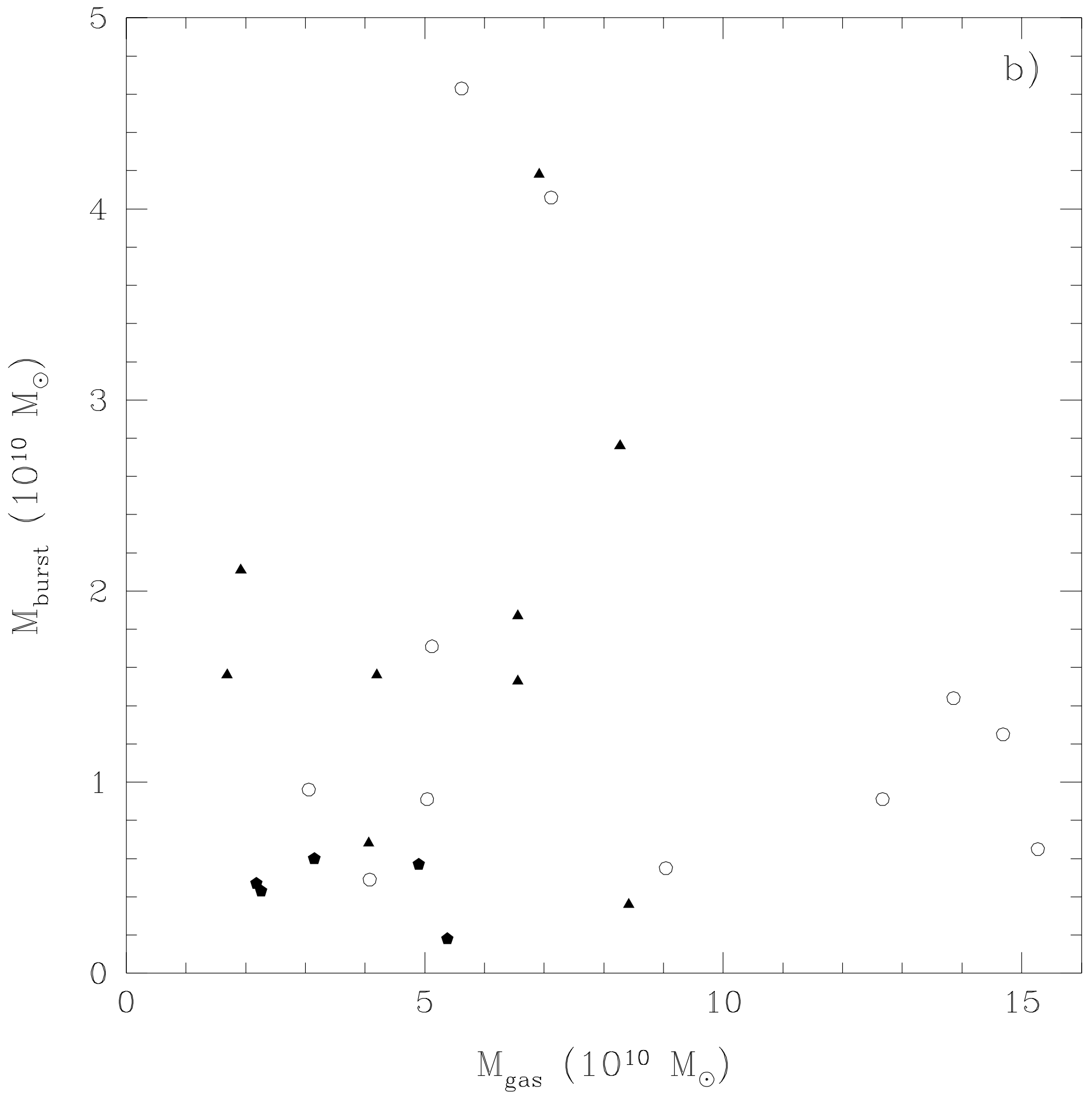




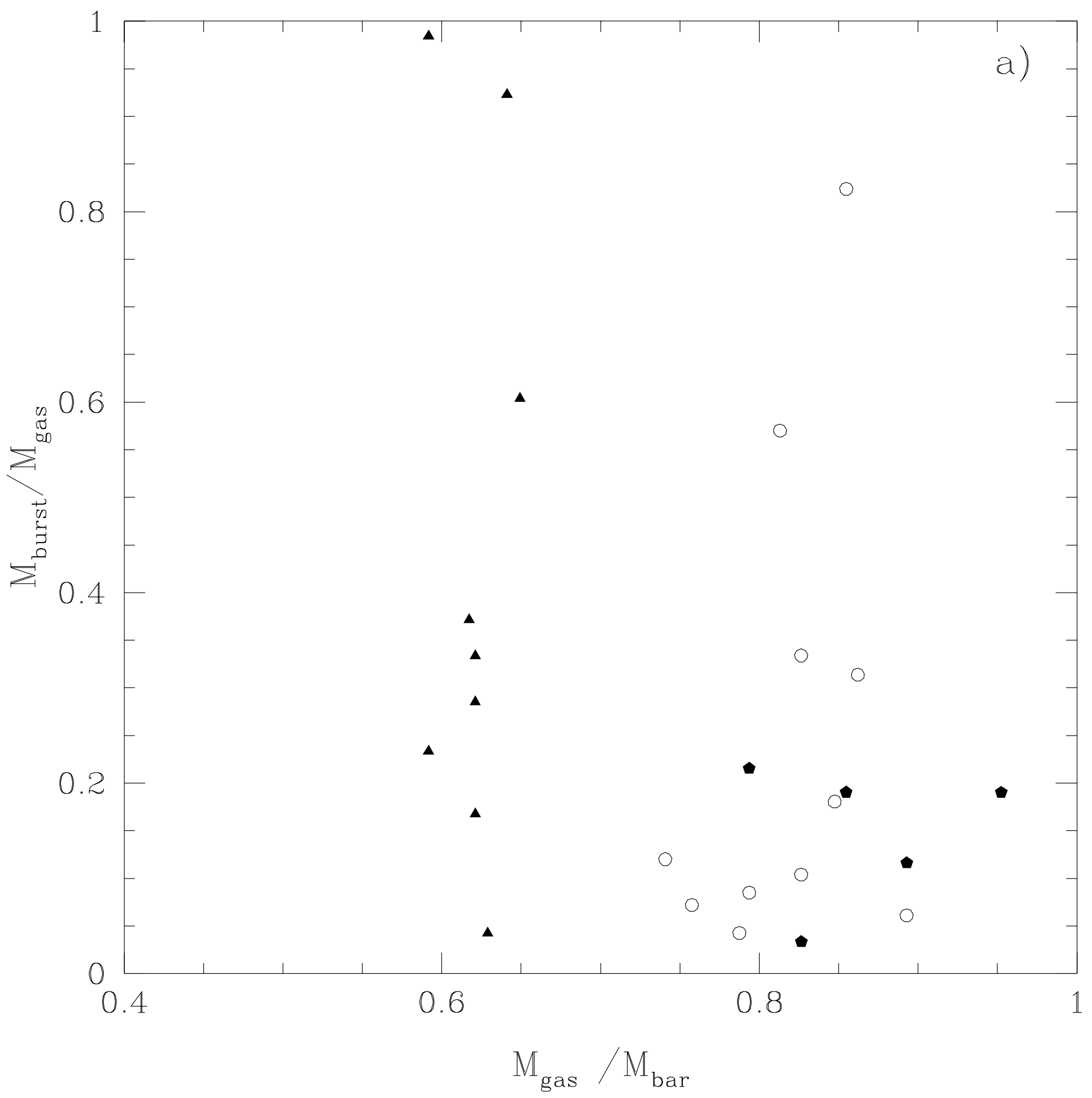




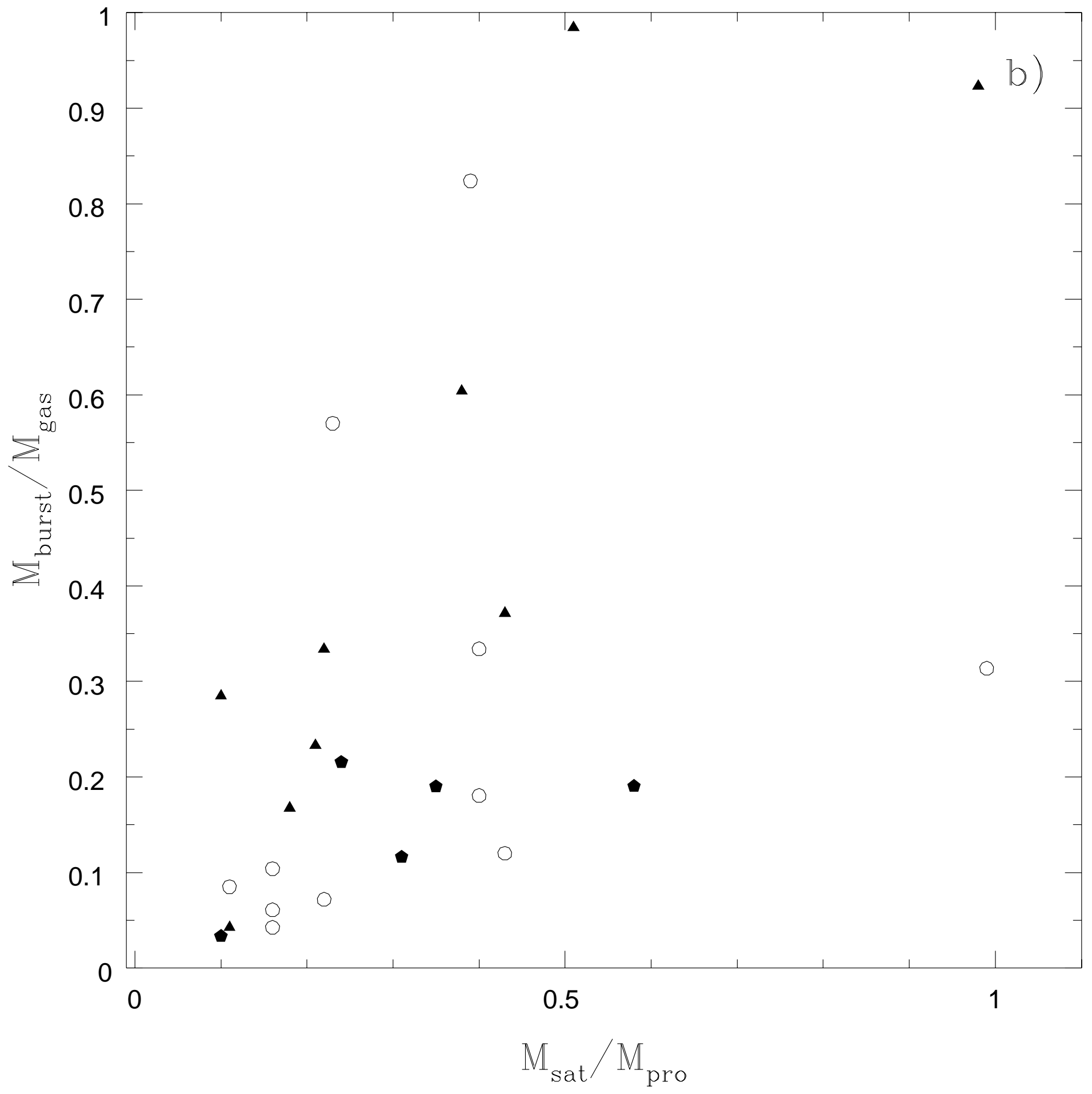




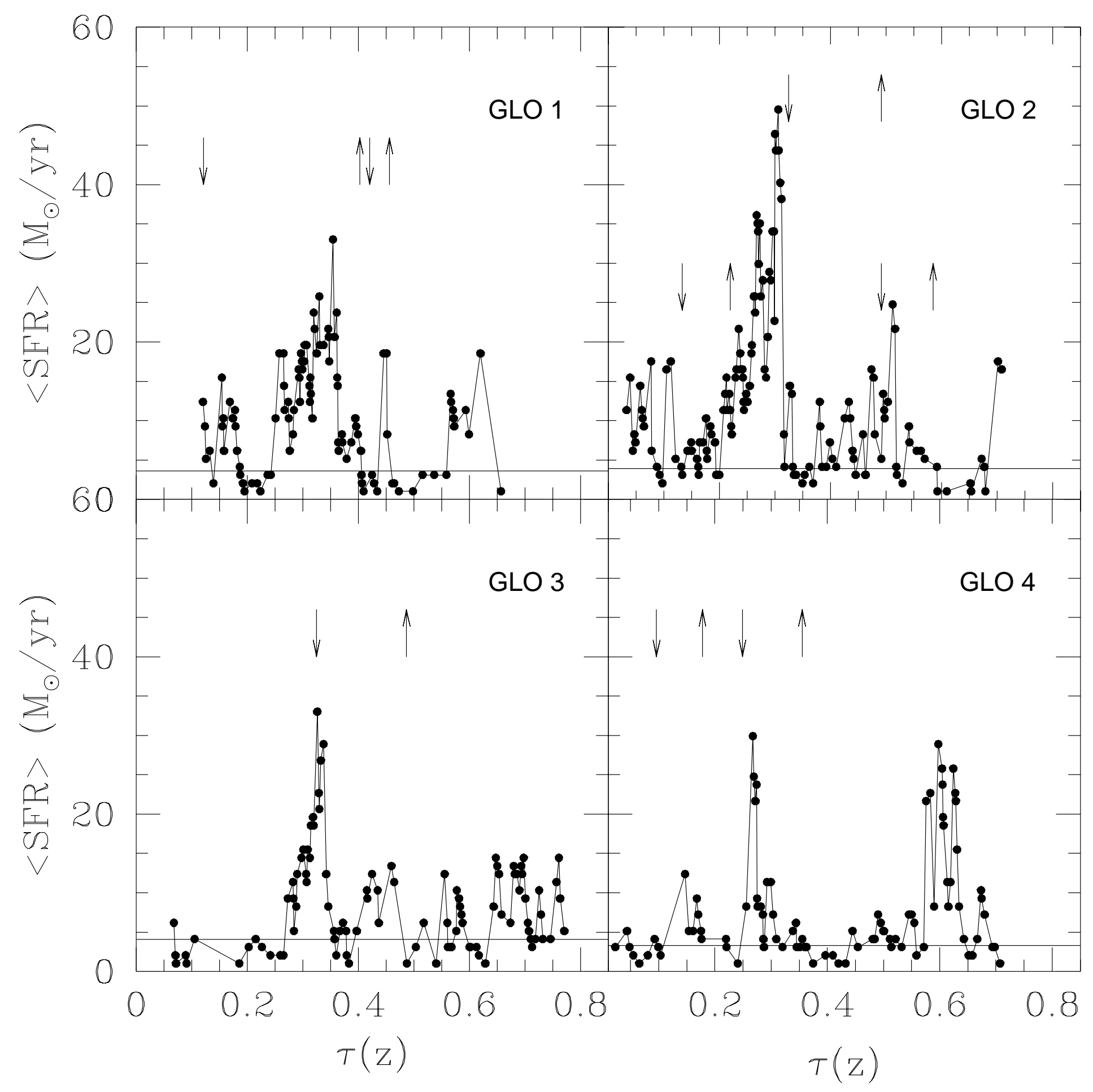




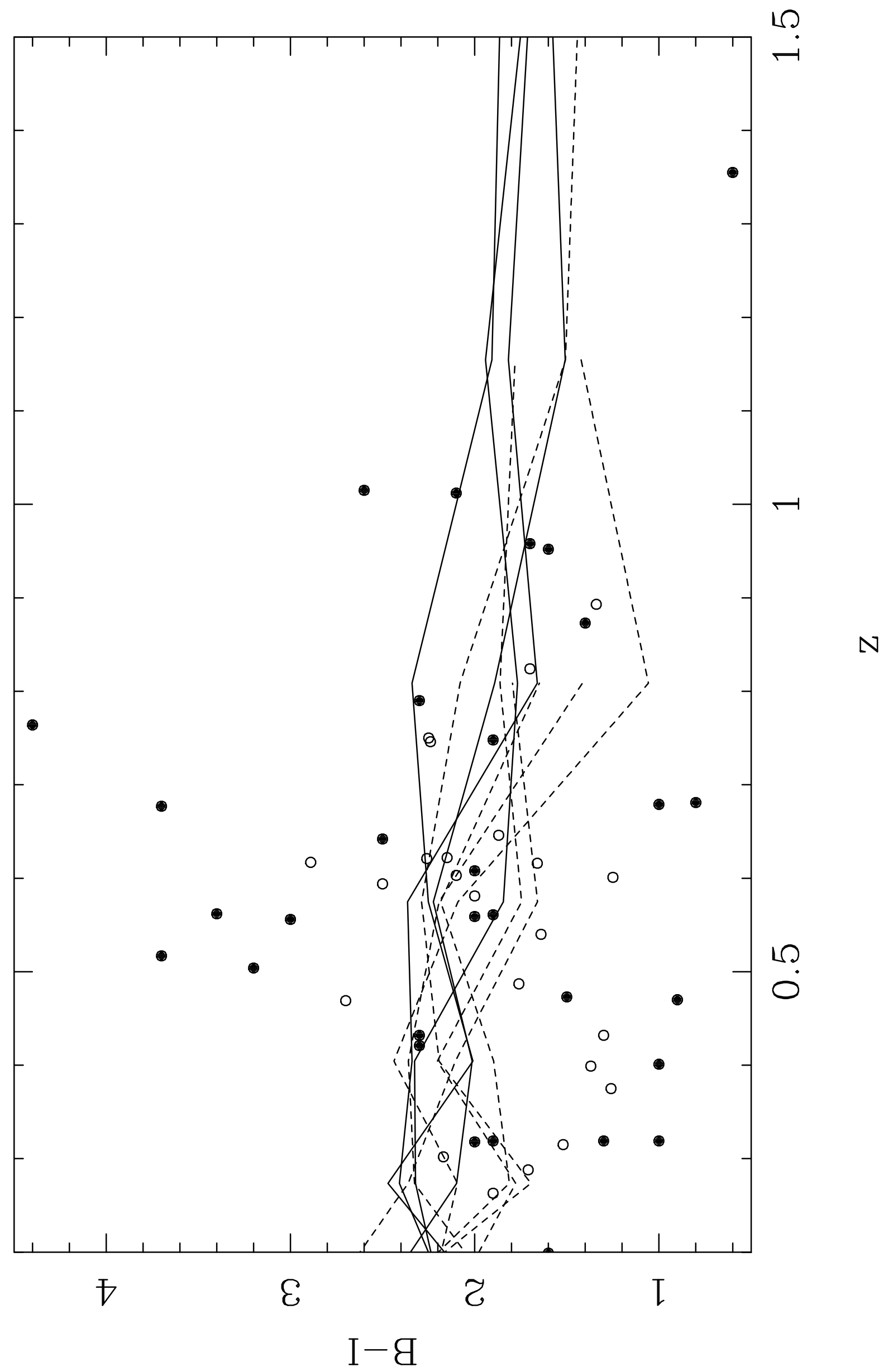




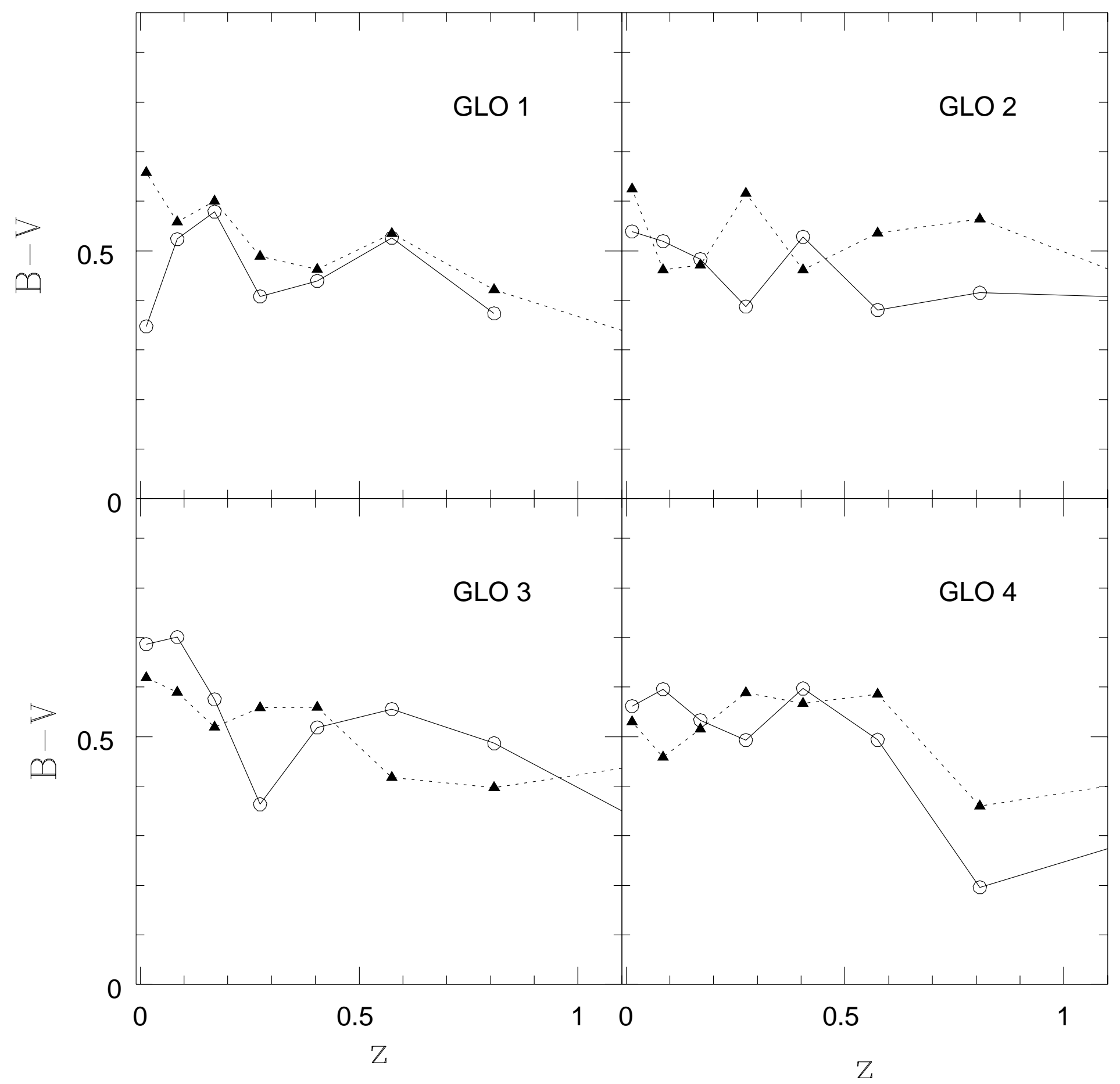




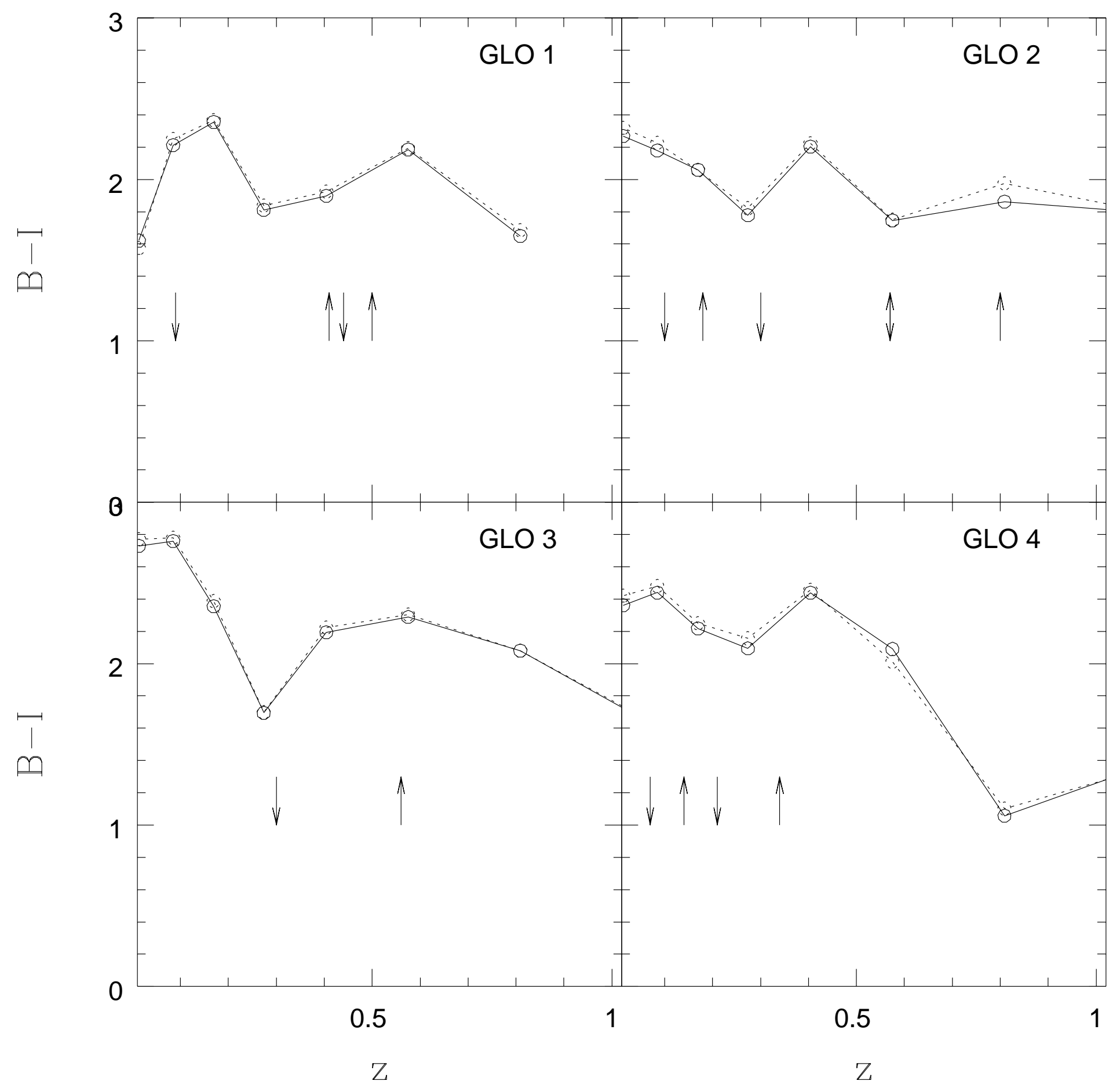



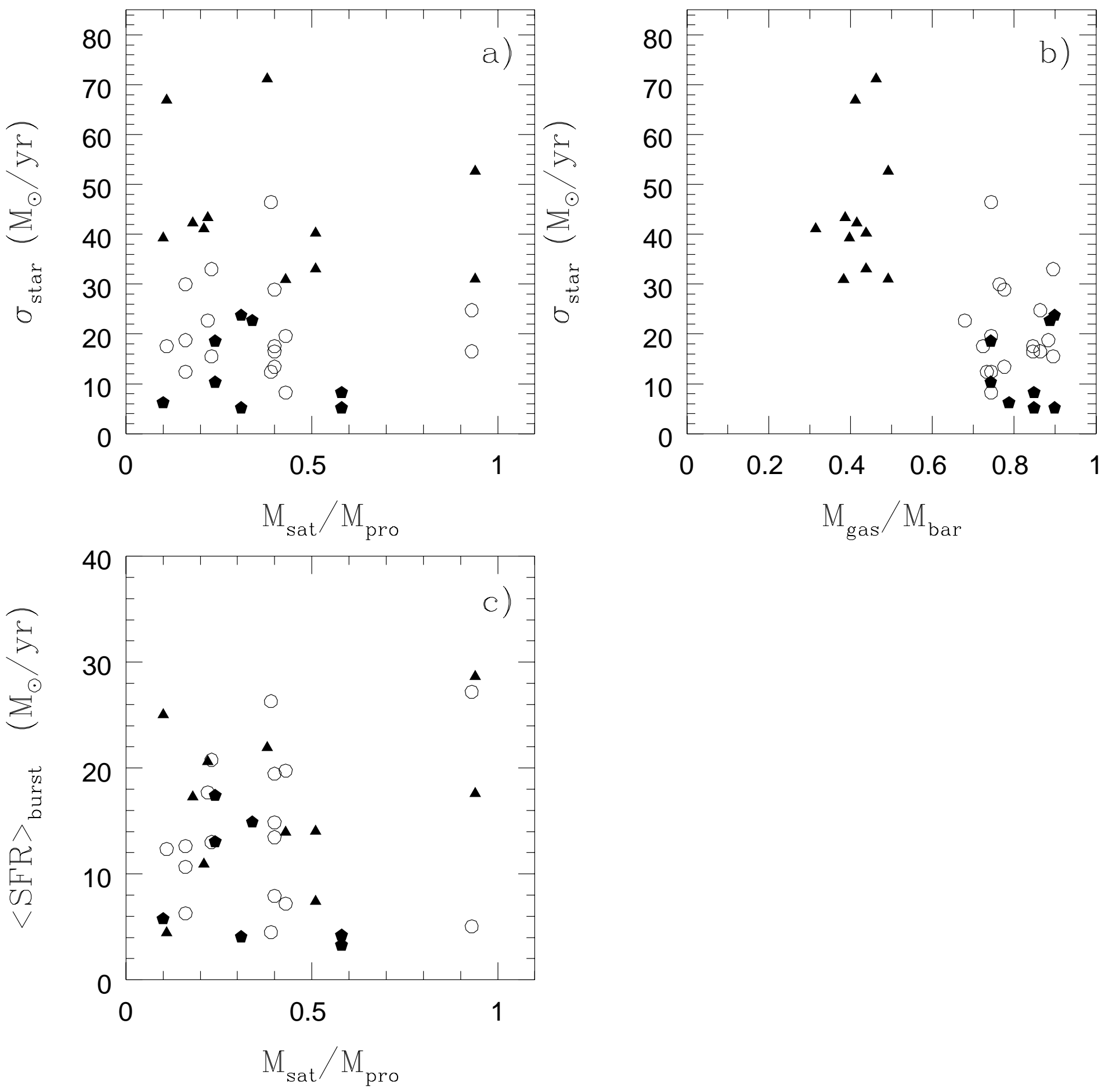


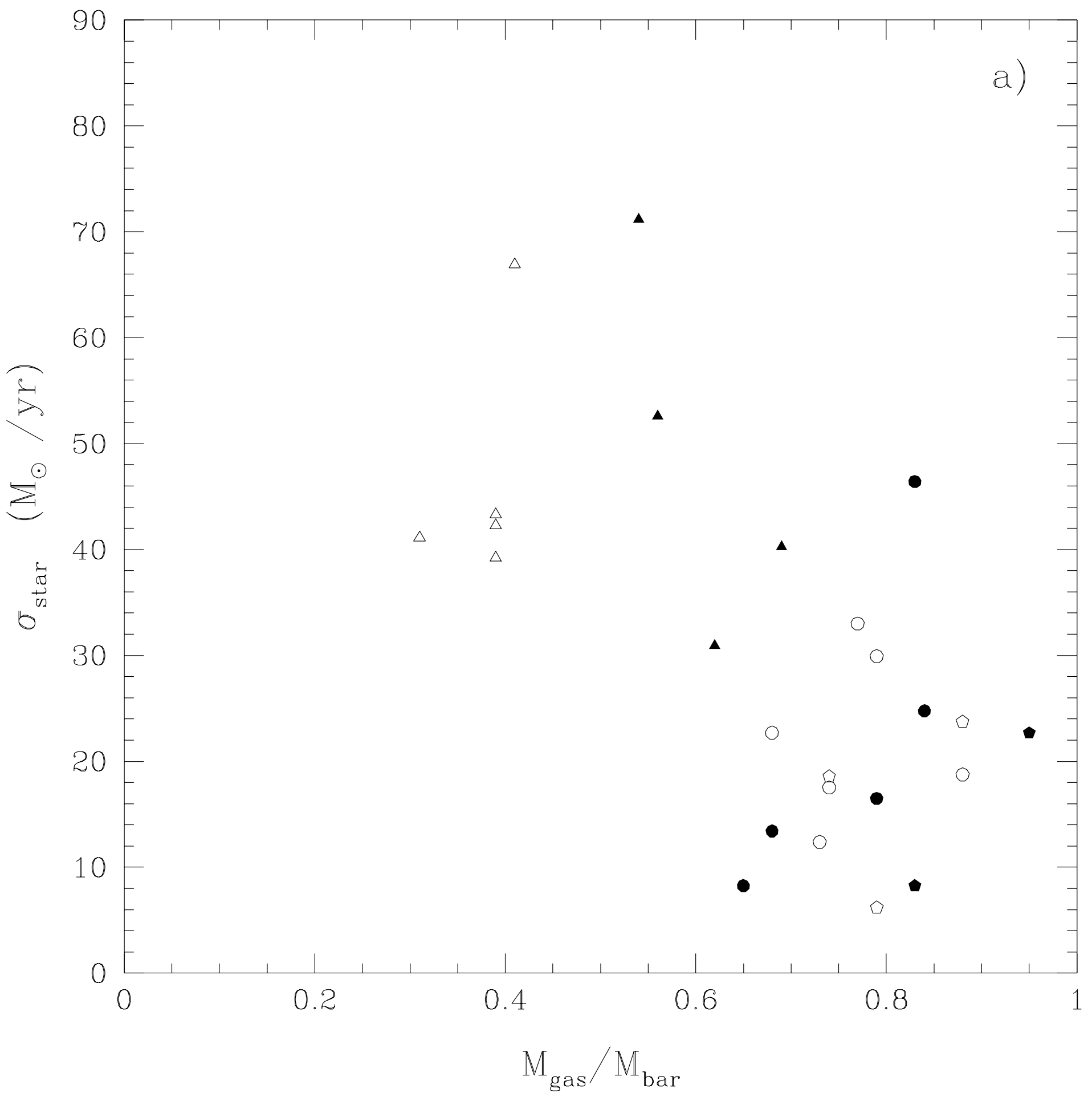




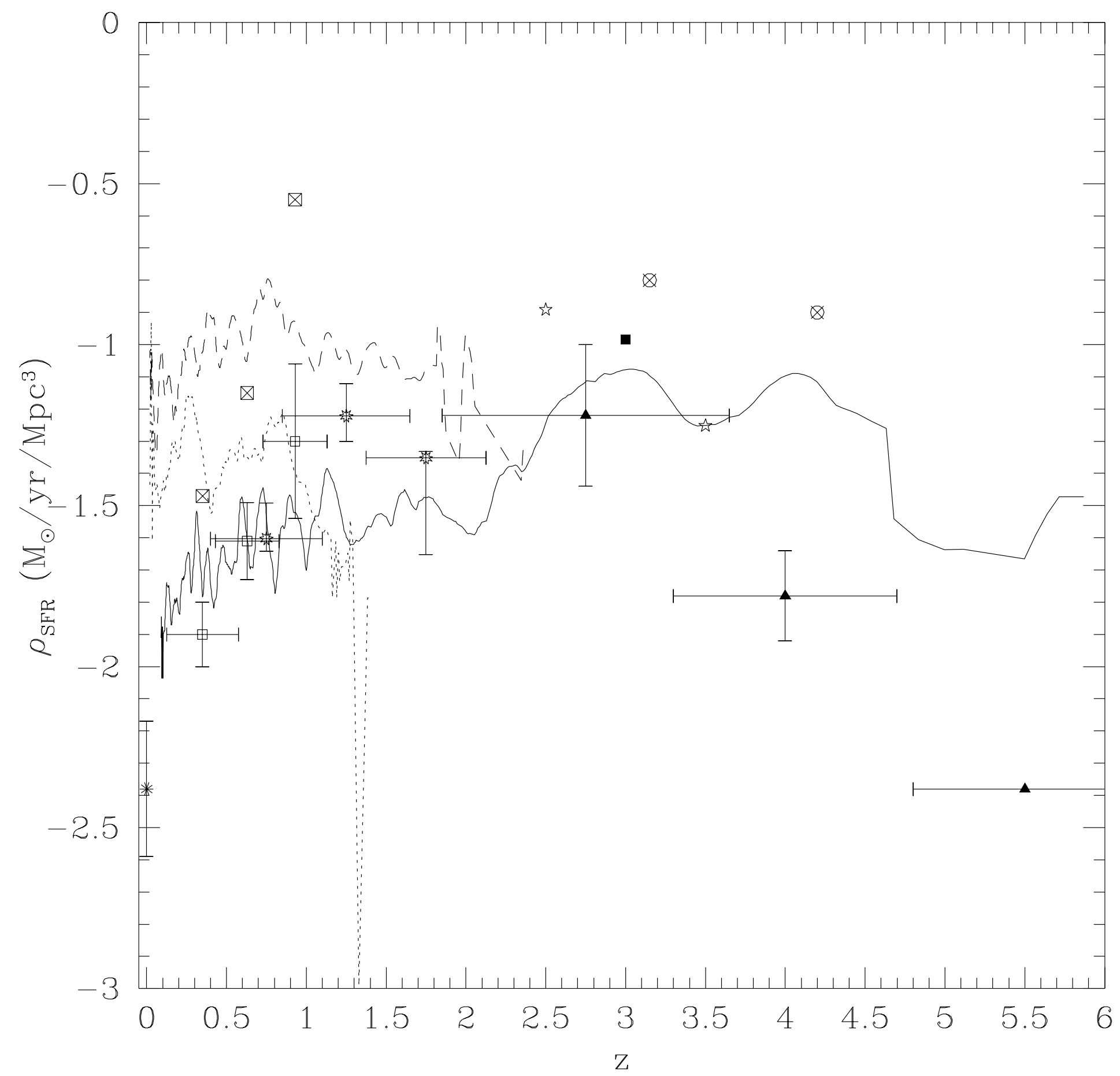

